\title{
The ataxia protein sacsin is required for integrin trafficking and synaptic organization
}

Lisa E.L. Romano ${ }^{1^{*}}$, Wen Yih Aw ${ }^{2^{\star}}$, Kathryn M. Hixson ${ }^{2^{\star}}$, Tatiana V. Novoselova ${ }^{1,3}$, Tammy M. Havener $^{2}$, Stefanie Howell ${ }^{2}$, Charlotte L Hall ${ }^{1}$, Lei Xing ${ }^{4}$, Josh Beri' ${ }^{5,6}$, Suran Nethisinghe ${ }^{1}$, Laura Perna $^{1}$, Abubakar Hatimy ${ }^{7}$, Ginevra Chioccioli Altadonna ${ }^{1}$, Lee M. Graves ${ }^{5,8}$, Laura E. Herring ${ }^{5,6}$, Anthony J. Hickey ${ }^{2}$, Konstantinos Thalassinos ${ }^{7,9}$, J. Paul Chapple ${ }^{1 \dagger}$, Justin M. Wolter ${ }^{2,4 \dagger}$

${ }^{1}$ William Harvey Research Institute, Barts and the London School of Medicine, Queen Mary University of London, London, EC1M 6BQ, United Kingdom.

${ }^{2}$ UNC Catalyst for Rare Diseases, Eshelman School of Pharmacy, The University of North Carolina at Chapel Hill, Chapel Hill, North Carolina, 27599, U.S.A.

${ }^{3}$ Department of Natural Sciences, Faculty of Science and Technology, Middlesex University London, NW4 4BT United Kingdom

${ }^{4}$ UNC Neuroscience Center, The University of North Carolina at Chapel Hill, Chapel Hill, North Carolina, 27599, U.S.A.

${ }^{5}$ Department of Pharmacology, University of North Carolina at Chapel Hill, Chapel Hill, NC 27599, USA

${ }^{6}$ UNC Michael Hooker Proteomics Center, University of North Carolina at Chapel Hill, Chapel Hill, NC 27599, USA

${ }^{7}$ Institute of Structural and Molecular Biology, Division of Biosciences, University College London, London, WC1E 6BT, United Kingdom.

${ }^{8}$ Lineberger Comprehensive Cancer Center, University of North Carolina at Chapel Hill, Chapel Hill, NC 27599, USA

${ }^{9}$ Institute of Structural and Molecular Biology, Birkbeck College, University of London, London, WC1E 7HX, United Kingdom

* These authors contributed equally to this work

$\dagger$ Co-corresponding authors

Correspondence:

Professor Paul Chapple

Centre for Endocrinology

William Harvey Research Institute

Barts and the London School of Medicine

Queen Mary University of London

Charterhouse Square

London EC1M 6BQ

United Kingdom

Tel: +44207882 6242

Email: j.p.chapple@qmul.ac.uk
Dr. Justin Wolter UNC Neuroscience Center University of North Carolina Chapel Hill, North Carolina 27599

U.S.A.

Tel:

Email: justin wolter@med.unc.edu 


\section{Abstract}

Autosomal recessive spastic ataxia of Charlevoix-Saguenay (ARSACS) is caused by mutations in SACS, which manifest as a childhood-onset cerebellar ataxia. Cellular ARSACS phenotypes include mitochondrial dysfunction, intermediate filament (IF) disorganization, and loss of Purkinje neurons. It is unclear how the loss of SACS causes these deficits, or why they manifest as cerebellar ataxia. We employed a multi-omics approach to characterize molecular and cellular deficiencies in SACS knockout (KO) cells. We identified alterations in microtubule structure and dynamics, protein trafficking, and mislocalization of synaptic and focal adhesion proteins. Targeting PTEN, a negative regulator of focal adhesions, rescued several cellular phenotypes in SACS KO cells. We found sacsin interacts with proteins implicated in vesicle transport, including HSP proteins, and interactions between structural and cell adhesion proteins were diminished in SACS KO cells. In all, this study suggests that trafficking and localization of synaptic adhesion proteins is a causal molecular deficiency in ARSACS. 


\section{Introduction}

ARSACS is a childhood-onset neurological disease characterized by pyramidal spasticity, cerebellar ataxia, and Purkinje cell loss, that is thought to have both neurodegenerative and neurodevelopmental components ${ }^{1}$. Initially thought to be localized to the Charlevoix-Saguenay region of Quebec, Canada, over 170 distinct mutations have now been identified, making ARSACS the second most common recessive cerebellar ataxia worldwide ${ }^{2}$.

The large size of SACS ( $520 \mathrm{kDa})$ has hampered biochemical and structural investigations into its function. SACS contains multiple domains that link it to molecular chaperone and protein quality control systems, including a ubiquitin like domain, three repetitive regions with some homology to the ATPase domain of Hsp90, and a J-protein domain, suggesting the potential to recruit Hsp70 chaperone proteins. Patient derived fibroblasts and the ARSACS mouse model possess consistent cellular phenotypes, including mitochondrial dysfunction, vimentin bundling, accumulation of non-phosphorylated neurofilament heavy, and altered firing properties of Purkinje neurons $s^{3-9}$. However, each of these deficits are found in a wide range of neurodegenerative diseases with diverse molecular causes ${ }^{10,11}$. Thus, the basic molecular function of sacsin, and why it's loss manifests as a cerebellar ataxia, remains unclear. 


\section{Results}

\section{Comprehensive proteomics of SACS KO cells}

To understand the molecular deficiencies in ARSACS, we deleted SACS using CRISPR/Cas9 in the SH-SY5Y human cell line (Extended Data Fig. 1a), which is widely used to model neurodegenerative diseases ${ }^{12}$. Consistent with ARSACS patient fibroblasts ${ }^{4}$ and SACS $^{(-/)}$ mice $^{7}, \mathrm{KO}$ cells had abnormal bundling and asymmetric partitioning of multiple IFs, including vimentin (Fig. 1a), neurofilament heavy, and peripherin (Extended Data Fig. 1b-e). However, whether abnormal IF structure plays a causal role in ARSACS pathology is unclear. As phosphorylation is a key modification controlling IF assembly and disassembly ${ }^{13}$, we performed quantitative proteomic and phosphoproteomic profiling of SACS KO cells (Supplementary Table 1). We identified changes in several proteins previously described in ARSACS patient fibroblasts, including vimentin, the mitochondrial protein ATP5J, and the autophagy regulated scaffold SQSMT1/p62 ${ }^{4}$ (Fig. 1b). Among the overabundant proteins were the tau-tubulin kinase 1 (TTBK1) and microtubule associated protein tau (MAPT) (Fig. 1c-f), which was hyperphosphorylated at several sites (Extended Data Fig. 1f, Fig. 1g,h, Supplementary Table 1). To assess the functional significance of specific phosphosites, we analyzed our data in light of a recent machine learning approach which estimated the effects of individual phosphosites on organism fitness ${ }^{14}$. This analysis identified several highly functional hypophosphorylated residues in vimentin, and the nuclear lamina IFs LMNA/B2, which is intriguing considering that ARSACS neurons have altered nuclear shape and positioning ${ }^{4}$ (Supplementary Table 1, Fig. 1i). Other hypophosphorylated proteins included the focal adhesion protein zyxin, and ataxin 2-like protein. In addition to tau, several other microtubule regulating proteins were hyperphosphorylated, including the primary cilia protein $A R L 3^{15}$, and the scaffold stathmin, which promotes microtubule assembly in a pS16 dependent fashion ${ }^{16}$.

Kinases are attractive drug targets ${ }^{17}$, but are typically lowly expressed and difficult to detect with standard proteomics. Therefore, we enriched for kinases using multiplexed kinase inhibitor beads, and performed quantitative mass-spectrometry ${ }^{18}$. The kinome was broadly altered in SACS KO cells (Extended Data Fig. 1g,h, Supplementary Table 1), with affected kinases spread among all kinase families (Fig. 1j). Interestingly, specific families were generally misexpressed in similar direction, for example the tyrosine kinase family (TK) members were generally downregulated, while CMGC family members were generally upregulated (Fig. 1j). The most overabundant kinase, BRSK2, and additional CAMK family members MARK1/2/3, all phosphorylate Ser262 in the microtubule binding domain of tau ${ }^{19,20}$ (Fig. 1k-m). Thr231, which is 
phosphorylated by DYRK1A, is also associated with the detachment of tau from microtubules $^{21,22}$. In all, there were 10 overexpressed kinases which phosphorylate tau at specific sites (Fig. 1n). In pathological settings, tau overabundance and hyperphosphorylation causes elevated microtubule stability, interferes with motor protein function, and alters axonal trafficking ${ }^{23-25}$. Combined with the altered phosphorylation of other microtubule related proteins (Fig. 1i), this data suggests that microtubule structure or function may be altered in ARSACS.

\section{Microtubule organization and dynamics are altered in SACS KO cells}

There is a bidirectional relationship between vimentin and microtubules, where vimentin filaments form along microtubule tracts, and can facilitate microtubule nucleation ${ }^{26}$. We found that cage-like vimentin bundles form around gamma-tubulin, a marker of the microtubule organizing center (MTOC), which is a central hub for microtubule nucleation and cargo transport $^{27}$ (Fig. 2a, Extended Data Fig. 2a). Acetylated alpha-tubulin, a microtubule stabilizing post-translational modification, was increased in SACS KO cells, without affecting total alphatubulin distribution or levels (Extended Data Fig. 2b, Fig. 2b,c). To assess microtubule dynamics we treated cells with the microtubule destabilizer nocodazole, and found enhanced microtubule polymerization following nocodazole washout (Fig. 2d,e). SACS KO cells also demonstrated increased microtubule polymerization and disordered movements as assessed by live cell imaging of the microtubule plus-end binding protein EB1:GFP (Fig. 2f, Extended Data Fig. 2e, Supplementary Video 1,2).

Mitochondrial movement in neurons is dependent on microtubules ${ }^{28}$. Tau overexpression and hyperphosphorylation can cause decreased mitochondria trafficking along microtubules $^{19,29,30}$, build-up of mitochondria around the MTOC ${ }^{31}$, and DRP1 mislocalization and reduced mitochondrial fission ${ }^{32,33}$. In ARSACS, mitochondria also accumulate around proximal dendrites ${ }^{6}$ and exhibit reduced DRP1 dependent fission ${ }^{3}$. We observed occlusion of mitochondria around vimentin bundles (Extended Data Fig. 2c), with no alterations in the actin cytoskeleton $^{4}$ (Extended Data Fig. 2d). To assess how these alterations affect mitochondria in neurons, we performed neuronal differentiation of SH-SY5Y cells ${ }^{34}$. WT and SACS KO cells expressed indistinguishable levels of neuronal markers, suggesting equal differentiation capacity (Extended Data Fig. 2f,g), but neurites were fewer and shorter in SACS KO cells (Extended Data Fig. 2f-i). SACS KO neurites also possessed fewer mitochondria (Extended Data Fig. 2j), and diminished mitochondrial movement (Extended Data Fig. 2k, Supplementary 
Video 3). Our proteomics data also identified hyperphosphorylation in several kinesin proteins, including multiple residues on KIF1A (Supplementary Table 1), which shuttle mitochondria along microtubule tracts ${ }^{35}$. In all, these results demonstrate altered microtubule structure, dynamics, and function in SACS KO cells, and are consistent with the microtubule stabilizing effects of tau and STMN1 pS16 hyperphosphorylation ${ }^{16}$.

\section{Focal adhesion organization and dynamics are disrupted in SACS KO cells}

To more systematically characterize our proteomic datasets, we performed gene ontology (GO) analysis (Fig. 3a,b, Supplementary Table 2). The top associated terms were related to 'focal adhesions', including 'integrin signaling', 'cell-matrix adhesions', and 'cadherin binding'. Focal adhesions are plasma membrane-associated macromolecular assemblies that physically link the intracellular cytoskeleton and extracellular matrix (ECM), and function as signaling nodes to regulate force dependent cellular processes ${ }^{36}$. Focal adhesions are composed of integrin receptors bridging the ECM with actin bundles, which interact with microtubules and IFs to coordinate dynamic regulation of focal adhesion structure ${ }^{37-39}$. In the brain, focal adhesions are critical for structural remodeling during axon growth and synapse formation and maintenance ${ }^{40}$.

We observed decreased focal adhesion number, area, and aspect ratio in SACS KO cells by immunolabelling for core focal adhesion components paxillin and vinculin, whose total levels were unaffected (Fig. 3c, Extended Data Fig. 3a-h, Supplementary Table 1). While paxillin is primarily localized at focal adhesions, it also is known to interact with the MTOC ${ }^{41}$, and we observed perinuclear accumulation of paxillin coinciding with the vimentin bundle (Extended Data Fig. 3a). Microtubules regulate vinculin localization to focal adhesions ${ }^{42}$, and we found reduced vinculin and vimentin dynamics in SACS KO cells using fluorescence recovery after photobleaching (FRAP) (Fig. 3d-f). We next removed cell bodies with hypotonic shock, leaving only the structural remnants of cell/ECM interactions, and again found reduced vinculin structures, suggesting that the mislocalization of adhesion proteins results in decreased cell/ECM interactions (Extended Data Fig. 3i-I). These findings were consistent in SACS KO HEK293 cells, which were generated using an alternate CRISPR/Cas9 genome editing strategy ${ }^{4}$ (Extended Data Fig. 3m-q). Our proteomics data also revealed changes in several integrin proteins (Fig. 3g). Localization of ITGAV to focal adhesions was lost in SACS KO cells (Fig. 3h), while ITGA6 was sequestered in the vimentin bundle (Fig. 3i). 


\section{Modulating PTEN-FAK signaling rescues cellular deficits in SACS KO cells}

Beyond providing structural support for cells, focal adhesions are enriched with many signaling proteins, which transmit signals from the extracellular milieu to effectors in the cytoplasm and nucleus. A master regulator of focal adhesion signaling is the focal adhesion kinase (FAK/PTK2) ${ }^{43}$, which regulates neuronal outgrowth and synapse formation by phosphorylating downstream effectors of focal adhesion signaling ${ }^{44}$ (Fig. 4a). Although total levels of FAK were unaltered, phosphorylated FAK (pFAK) was significantly reduced in SACS KO cells (Fig. 4b, Extended Data Fig. 4a,b). Similarly, JNK and paxillin were also hypophosphorylated, while total levels of these proteins were unaffected (Fig. 4b, Extended Data Fig. 4c-g, Supplementary Table 1). Localization of pFAK to focal adhesions was also decreased SACS KO cells (Fig. 4c). We next considered the mechanism by which FAK signaling is suppressed in SACS KO cells. The phosphatase PTEN, which dephosphorylates FAK and negatively regulates FAK activity ${ }^{45}$, was elevated in SACS KO cells (Fig. 4b, Extended Data Fig. 4h). To investigate whether increased PTEN is a general consequence of IF disorganization, we treated WT SH-SY5Y cells with simvastin ${ }^{46}$, which induced bundling and perinuclear accumulation of vimentin, but did not affect PTEN levels (Extended Data Fig. 4i-k). Conversely, reducing PTEN to WT levels in SACS KO cells (Extended Data Fig. 4I,m) increased pFAK (Fig. 4d), reduced the frequency of perinuclear vimentin accumulation (Fig. 4e,f), increased the number of focal adhesions (Fig. 4e,g), and restored migration deficits of SACS KO cells (Extended Data Fig. 4n,o). Together these results indicate that increased PTEN activity contributes, at least in part, to the IF and focal adhesion phenotypes in SACS KO cells. Furthermore, our data suggests that modulating this pathway may reduce molecular deficits associated with ARSACS.

\section{Membrane bound synaptic adhesion molecules are mislocalized in SACS KO cells}

Focal adhesions act as signal transduction hubs to integrate information from the outside of the cell to the inside. Some focal adhesion proteins, including paxillin and zyxin (Fig. 1i), can shuttle to the nucleus and function as transcriptional coregulators in a phosphorylation dependent manner ${ }^{47,48}$. GO term analysis of hyperphosphorylated proteins identified several terms related to transcriptional processes, including nuclear matrix, RNA binding, and transcriptional coregulator activity (Extended Data Fig. 5a, Supplementary Table 2). Therefore, 
we performed RNA-seq in neuronally differentiated SH-SY5Y cells to determine how structural changes affect the neuronal transcriptome (Extended Data Fig. 5b, Supplementary Table 3). We found 876 differentially expressed genes (FDR<0.05, f.c. $-/+0.4$ ), suggesting the loss of sacsin has profound effects on the transcriptome (Extended Data Fig. 5b). GO term analysis identified multiple terms related to membrane proteins and neuronal synapses, including 'post-synaptic membrane' and 'axon terminus' (Extended Data Fig. 5c). Protein interaction mapping ${ }^{49}$ revealed altered expression of multiple ECM proteins, integrins, and regulators of integrin activation (Extended Data Fig. 5d), suggesting that membrane dependent signaling cascades affect the transcription of functionally related genes.

Cell surface proteins are frequently underrepresented in proteomics experiments due to low expression and biochemical properties ${ }^{50}$. While $26 \%$ of all genes identified by RNAseq were detected by proteomics experiments, only $11 \%$ of differentially expressed genes (DEGs) were detected by proteomics (Extended Data Fig. 5e), which is in agreement with GO term analyses suggesting alterations in membrane dependent processes. Therefore to enrich for surface proteins, we incubated live cells with biotin, which labels cellular and exosomal membrane/surface proteins, followed by neutravidin purification and analysis by quantitative mass-spectrometry ${ }^{51}$ (Fig. 5a, Supplementary Table 1). This approach identified an additional 870 proteins not in our proteomic dataset (Extended Data Fig. 5f, Fig. 5b). Proteins altered KO cells included several signaling receptors (FGFR1, LRP4, NRP2), and GTP binding proteins involved in signal transduction (GNG2, GNG8). The most overabundant membrane protein was neurofascin (NFASC), a neuronal adhesion protein that has been linked to movement disorders and cerebellar ataxia ${ }^{52,53}$ (Fig. 5c). We next compared membrane proteins found in both proteomic and surfaceome datasets, reasoning that conflicting levels between the surface and total protein levels could reflect improper membrane recycling, precocious membrane localization, or deficits in membrane-bound trafficking. Many proteins with altered surface levels showed no, or even opposing change in total protein levels (Fig. 5d). Among the most mislocalized proteins were synaptic adhesion proteins, including multiple integrins (ITGA1, ITGB1, ITGA3), neuronal cell adhesion molecules (L1CAM, NRCAM, CNTN1, LSAMP), the focal adhesion regulator RET/GFRA3 heterodimer, the microtubule binding protein DCX, and AHNAK, a 700 kDa scaffolding protein with diverse yet poorly understood function ${ }^{54}$ (Fig. 5d).

GO term analysis of proteins with altered surface levels suggested deficits in processes related to vesicle packaging and transport (Fig. 5e). These included eight exosomal Rab proteins, which were increased in the surfaceome and not affected at the total protein level 
(Extended Data Fig. 5g, Supplementary Table 1). Rabs are a diverse family of GTPases that coordinate multiple aspects of membrane protein trafficking, including focal adhesion turnover, and integrin endo/exocytosis ${ }^{55}$. Specific Rabs also regulate trafficking between the Golgi and the endosomal network (RAB8A, RAB10), bidirectional Golgi/ endoplasmic reticulum (ER) trafficking (RAB2A, RAB18), and EGFR internalization (RAB7A) ${ }^{56,57}$. Kinome profiling also identified multiple regulators of Rab activity and trafficking, including PIK3R4 and PIK3C3, which regulate PTEN activity through localization to vesicles in a microtubule dependent fashion ${ }^{58}$ (Fig. 1j, Supplementary Table 1).

To assess trafficking and localization deficits independent of changes in protein level or phosphorylation, we investigated the localization of the ECM protein fibronectin, which is processed and packaged into vesicles in the ER and Golgi ${ }^{59}$, and trafficked to the cell periphery along microtubules ${ }^{60}$. In WT HEK293 cells fibronectin puncta were organized in 'chains', which appear collapsed around the vimentin bundle in SACS KO cells (Fig. 5f). Staining for the ER marker KDEL suggested that fibronectin is retained in the ER in HEK293 and SH-SY5Y SACS KO cells (Fig. 5g, Extended Data Fig. 5h), suggesting that membrane bound trafficking is substantially affected in SACS KO cells.

We next used Ingenuity Pathway Analysis (IPA) to assess whether the misregulated cell surface proteins are associated with any pathological conditions. Resoundingly, the terms were associated with disease traits reminiscent of ARSACS, including movement disorders, neurodegeneration, and progressive neurological disorder (Fig. 5h). Notably, three of the most mislocalized proteins, NFASC, NRCAM, and CNTN1, form molecular complexes that are important for axon guidance ${ }^{61}$, maintenance of synapses by astrocytes ${ }^{62}$, and interactions between Purkinje neuron axons and glia $^{63}$. KO mice or humans which harbor mutations in each of these genes develop cerebellar ataxias with features that resemble ARSACS (see Discussion).

\section{Cell adhesion proteins are mislocalized in ARSACS mice}

We next looked for evidence of changes in protein localization in $S A C S^{(-/)}$mice at P120, which is during the initial period of Purkinje neuron loss $^{7}$ (Fig. 6a). ITGA1, which was among the most mislocalized proteins in SACS KO cells (Fig. $5 \mathrm{~d}$ ), is normally localized along Purkinje axon tracts in unaffected mice, but in $S A C S^{(-)}$mice ITGA1 accumulated in the soma, dendritic trunk, and axonal swellings (Extended Data Fig. 6a, Fig. 6b). NFASC is normally enriched along the 
axon initial segment of Purkinje neurons where it contributes to action potential initiation ${ }^{64}$. Sacs $^{(--)}$Purkinje neurons showed increased NFASC intensity, as well as expanded localization around the circumference of the soma (Fig. 6c, Extended Data Fig. 6b). Purkinje neurons synapse onto neurons in the deep cerebellar nucleus (DCN), which is the primary output hub of the cerebellum. We observed striking loss of Purkinje neurons synapses onto DCN neurons in Sacs $^{(--)}$mice (Fig. 6d), which may be present at presymptomatic stages ${ }^{9}$. Colocalization between the presynaptic marker SYN1 and paxillin was only evident on DCN neurons in unaffected mice, but showed large extracellular accumulations in $S A C S^{(-)}$mice (Extended Data Fig. 6b). We also observed abnormal axon termini from layer $\mathrm{V}$ cortical motor projection neurons in the spinal cord (Extended Data Fig. 7a,b), as well as smaller axonal bundles in $S A C S^{(--)}$mice (Extended Data Fig. 7c).

\section{The loss of sacsin disrupts protein-protein interactions}

To identify how the loss of SACS causes abnormal protein trafficking, we performed quantitative label-free mass spectrometry of endogenous sacsin co-immunoprecipitated (co-IP) from WT SH-SY5Y cells. KO cells were also used to control for non-specific protein pulldown. Our analysis identified 90 proteins as putative Sacsin interactors, including vimentin and vinculin (Supplementary Table 4). Immunofluorescence revealed sacsin puncta in and around vinculin positive focal adhesions (Extended Data Fig. 8a,b), and in close proximity to vimentin structures, with sacsin often being between them (Fig. 7a). Reciprocal co-IP experiments confirmed interactions between sacsin, vimentin and vinculin, but the interaction between vimentin and vinculin was dramatically reduced in SACS KO cells (Fig. 7b). NFASC has been reported to interact with vimentin ${ }^{65}$, leading us to wonder whether NFASC may also interact with focal adhesion proteins. Co-IP experiments identified an interaction between NFASC and vinculin, which was dramatically reduced in SACS KO cells (Fig. 7c), suggesting that sacsin promotes the formation and/or stabilization of adhesion protein interactions.

To identify central proteins which may explain the cellular phenotypes in SACS KO cells, we performed STRING network analysis ${ }^{49}$. We considered all proteins altered in any dataset, and assessed only high confidence physical or regulatory interactions. K-means clustering of network interactions identified three clusters, each of which highlight complementary pathways by which sacsin contributes to cell structure and signaling (Fig. 7d). Central to cluster 1 is the interaction between sacsin and IF proteins, which interact with a variety of cell surface receptors. Combined with our biochemical experiments, this suggests that the loss of sacsin 
leads to improper localization of adhesion proteins to the plasma membrane, through decreased protein interactions between IFs, adaptors, and adhesion proteins. PTEN regulates several of these proteins, suggesting additional targets beyond FAK which may contribute to SACS KO phenotypes. The network also highlighted the microtubule associated kinase MAST1 (Fig. 1j, increased expression), which stabilizes $\mathrm{PTEN}^{66}$, and is protected from proteasomal degradation by the SACS interactor HSP9OB $1^{67}$.

Cluster 2 is composed of the interaction between sacsin, chaperone network proteins, and microtubules, which in concert regulate membrane protein processing, trafficking, and localization $^{68}$. Multiple HSP chaperones were part of the sacsin interactome (Fig. 7d), including the marker of ER stress HSPA5/BIP, and several HSP90 proteins, which can stabilize FAK, modulate cell migration ${ }^{69}$, and regulate microtubules ${ }^{70}$. Recent evidence suggests HSP90 is essential for microtubule acetylation ${ }^{71}$, suggesting that the loss of sacsin may alter microtubule stability via HSP proteins (Fig. 2d,e). HSP proteins also regulate Rab proteins ${ }^{72}$ (cluster 3), which have diverse roles in vesicular trafficking, including PTEN and EGFR trafficking ${ }^{73}$. GO term analysis revealed that $65 \%$ of sacsin interacting proteins are involved in exosome related processes, with additional interactors being implicated in unfolded protein binding (HSPS) and focal adhesions (Extended Data Fig. 8c). In all, these results suggest that sacsin plays a critical role in functionally bridging protein quality control systems, microtubule dependent vesicular transport, and membrane localization of adhesion proteins. 


\section{Discussion}

This study identifies sacsin as a critical regulator of multiple aspects of cellular structure, including IF architecture, microtubules, protein trafficking, and focal adhesions. The complex and intertwined relationships between these processes complicates our understanding of their precise pathophysiological relevance, but our study raises some intriguing possibilities. Sacsin possesses a functional $\mathrm{J}$ domain, which interacts with HSP70 chaperone proteins ${ }^{74,75}$ (Fig. 7d). HSP proteins play a role in ubiquitin dependent turnover of $\mathrm{IFs}^{76}$, and neurofilament bundling in ARSACS neurons can be rescued by HSP expression ${ }^{5}$. Sacsin also possesses an ATPase domain with homology to HSP90 proteins. The sacsin interactor HSP90B1 stabilizes FAK ${ }^{69}$, suggesting that restoring FAK signaling may rescue IF structure through HSP activity (Fig. 4,7d). It is also possible that sacsin transiently interacts with HSP90 regulated kinases, such as $\mathrm{FAK}^{69}$, and has a more direct role at focal adhesions. HSP70/90 complexes bind to microtubules in an acetylation dependent fashion ${ }^{77}$, and interact with hyperphosphorylated tau to increase tau's interaction with microtubules ${ }^{78}$. Since HSPs are known to regulate all of the protein clusters with deficits in SACS KO cells (Fig. 7d), we hypothesize that the interaction between HSPs and sacsin may be an especially critical interaction that is lost in ARSACS. Furthermore, as illustrated by sacsin's mediation of the interaction between IFs and focal adhesions, changes in additional as yet uncharacterized protein-protein interactions may explain specific ARSACS phenotypes, such as disrupted autophagy, nuclear morphology, and aberrant localization of mitochondria.

Why do mutations in sacsin, which is expressed throughout the brain, present as a cerebellar ataxia? Proteins whose abundance or localization are altered in SACS KO cells, and which also cause cerebellar ataxia, could suggest a causal molecular deficiency in ARSACS. The interactions between NFASC, NRCAM, and CNTN1 are critical for brain development, and mutation of each causes phenotypes reminiscent of ARSACS. Cntn1 KO mice have deficits in axon guidance and develop cerebellar ataxia ${ }^{79}$. Nrcam KO mice have phenotypes only in lobules $4 / 5$ of the cerebellar vermis ${ }^{80}$, which are also specifically affected in ARSACS ${ }^{7,9,81}$. Lastly, human mutations in NFASC which selectively remove the $155 \mathrm{kD}$ glial isoform cause congenital hypotonia, demyelinating neuropathy (as in ARSACS) and severe motor coordination defects $^{52}$, while mutations of the neuron specific $186 \mathrm{kD}$ NFASC isoform cause cerebellar ataxia $^{53}$. These convergent phenotypes lead us to hypothesize that improper localization of synaptic cell adhesion molecules is a causal molecular deficiency in ARSACS. Determining the mechanism by which sacsin controls the localization and function of these proteins throughout 
bioRxiv preprint doi: https://doi.org/10.1101/2021.08.20.456807; this version posted September 2, 2021. The copyright holder for this preprint

(which was not certified by peer review) is the author/funder, who has granted bioRxiv a license to display the preprint in perpetuity. It is made available under aCC-BY-NC-ND 4.0 International license.

disease progression will be critical to our understanding of ARSACS pathophysiology, and developing rationally designed therapeutic strategies. 


\section{ACKNOWLEDGEMENTS}

We express our gratitude to Sonia Gobeil and the ARSACS patient community. This work was supported by grants to J.P.C and J.M.W. from the Fondation de l'Ataxie Charlevoix-Saguenay. We thank Thomas Sterns Karim Gilbert, Natalie Barker, and Dennis Goldfarb for technical assistance. We thank Dr. Stefan Strack for generously providing ARSACS mice. JPC was supported by the BBSRC (BB/R003335/1) and Ataxia UK. JMW was supported by grants from the National Institute for Child Health and Human Development (NICHD; T32HD040127) and a Pfizer-NCBiotech Postdoctoral Fellowship in Gene Therapy. LMG is supported by NIH R01 GM138520. The UNC Catalyst for Rare Diseases gratefully acknowledges the support of the Eshelman Institute for Innovation. This research is based in part upon work conducted using the UNC Proteomics Core Facility, which is supported in part by P30 CA016086 Cancer Center Core Support Grant to the UNC Lineberger Comprehensive Cancer Center. Confocal microscopy was performed using a microscope funded by Barts Charity (MGU0293). The UNC microscopy core is supported by the NICHD (P50HD103573). 


\section{AUTHOR CONTRIBUTIONS}

JMW and JPC conceived of the study. JMW, JPC, and LELR analyzed data and prepared the manuscript. SN, TMH, and JMW created SACS KO neuroblastoma lines. LMG aided in experimental design and provided reagents for proteomics experiments. AJH and KT provided reagents and experimental oversight. WYA, KMH, LX, and JMW performed histology. $\mathrm{SH}, \mathrm{TH}$, and WA managed the mouse colony. $\mathrm{KMH}, \mathrm{TMH}, \mathrm{SH}, \mathrm{JB}, \mathrm{LEH}$, and JMW performed quantitative proteomics experiments. TVN and CLH performed RNAseq, KH and JMW analyzed the data. TVN and CLH performed the SACS interactome experiments. AH and KT performed co-IP MS experiments. LELR, WYA, KH, TVN, TMH, SH, LP, JMW, and GCA performed cell biology experiments. 


\section{REFERENCES}

1 Vermeer, S., van de Warrenburg, B. P., Kamsteeg, E. J., Brais, B. \& Synofzik, M. in GeneReviews((R)) (eds M. P. Adam et al.) (1993).

2 Synofzik, M. et al. Autosomal recessive spastic ataxia of Charlevoix Saguenay (ARSACS): expanding the genetic, clinical and imaging spectrum. Orphanet journal of rare diseases 8, 41, doi:10.1186/1750-1172-8-41 (2013).

3 Bradshaw, T. Y. et al. A reduction in Drp1-mediated fission compromises mitochondrial health in autosomal recessive spastic ataxia of Charlevoix Saguenay. Human molecular genetics 25, 3232-3244, doi:10.1093/hmg/ddw173 (2016).

4 Duncan, E. J. et al. Altered organization of the intermediate filament cytoskeleton and relocalization of proteostasis modulators in cells lacking the ataxia protein sacsin. Human molecular genetics 26, 3130-3143, doi:10.1093/hmg/ddx197 (2017).

5 Gentil, B. J. et al. Sacsin, mutated in the ataxia ARSACS, regulates intermediate filament assembly and dynamics. The FASEB journal : official publication of the Federation of American Societies for Experimental Biology 33, 2982-2994, doi:10.1096/fj.201801556R (2019).

6 Girard, M. et al. Mitochondrial dysfunction and Purkinje cell loss in autosomal recessive spastic ataxia of Charlevoix-Saguenay (ARSACS). Proceedings of the National Academy of Sciences of the United States of America 109, 1661-1666, doi:10.1073/pnas.1113166109 (2012).

$7 \quad$ Lariviere, R. et al. Sacs knockout mice present pathophysiological defects underlying autosomal recessive spastic ataxia of Charlevoix-Saguenay. Human molecular genetics 24, 727-739, doi:10.1093/hmg/ddu491 (2015).

8 Morani, F. et al. Functional Transcriptome Analysis in ARSACS KO Cell Model Reveals a Role of Sacsin in Autophagy. Scientific reports 9, 11878, doi:10.1038/s41598-01948047-x (2019).

9 Ady, V. et al. Altered synaptic and firing properties of cerebellar Purkinje cells in a mouse model of ARSACS. The Journal of physiology 596, 4253-4267, doi:10.1113/JP275902 (2018).

10 Stanga, S., Caretto, A., Boido, M. \& Vercelli, A. Mitochondrial Dysfunctions: A Red Thread across Neurodegenerative Diseases. International journal of molecular sciences 21, doi:10.3390/ijms21103719 (2020).

11 Didonna, A. \& Opal, P. The role of neurofilament aggregation in neurodegeneration: lessons from rare inherited neurological disorders. Molecular neurodegeneration 14, 19, doi:10.1186/s13024-019-0318-4 (2019).

12 Xicoy, H., Wieringa, B. \& Martens, G. J. The SH-SY5Y cell line in Parkinson's disease research: a systematic review. Molecular neurodegeneration 12, 10, doi:10.1186/s13024-017-0149-0 (2017).

13 Snider, N. T. \& Omary, M. B. Post-translational modifications of intermediate filament proteins: mechanisms and functions. Nature reviews. Molecular cell biology 15, 163-177, doi:10.1038/nrm3753 (2014).

14 Ochoa, D. et al. The functional landscape of the human phosphoproteome. Nature biotechnology 38, 365-373, doi:10.1038/s41587-019-0344-3 (2020). 
15 Zhou, C., Cunningham, L., Marcus, A. I., Li, Y. \& Kahn, R. A. Arl2 and Arl3 regulate different microtubule-dependent processes. Molecular biology of the cell 17, 2476-2487, doi:10.1091/mbc.e05-10-0929 (2006).

16 Di Paolo, G., Antonsson, B., Kassel, D., Riederer, B. M. \& Grenningloh, G. Phosphorylation regulates the microtubule-destabilizing activity of stathmin and its interaction with tubulin. FEBS letters 416, 149-152, doi:10.1016/s0014-5793(97)01188-5 (1997).

17 Krahn, A. I. et al. Defining the Neural Kinome: Strategies and Opportunities for Small Molecule Drug Discovery to Target Neurodegenerative Diseases. ACS Chem Neurosci 11, 1871-1886, doi:10.1021/acschemneuro.0c00176 (2020).

18 Cooper, M. J. et al. Application of multiplexed kinase inhibitor beads to study kinome adaptations in drug-resistant leukemia. PLoS One 8, e66755, doi:10.1371/journal.pone.0066755 (2013).

19 Ando, K. et al. Stabilization of Microtubule-Unbound Tau via Tau Phosphorylation at Ser262/356 by Par-1/MARK Contributes to Augmentation of AD-Related Phosphorylation and Abeta42-Induced Tau Toxicity. PLoS genetics 12, e1005917, doi:10.1371/journal.pgen.1005917 (2016).

20 Kishi, M., Pan, Y. A., Crump, J. G. \& Sanes, J. R. Mammalian SAD kinases are required for neuronal polarization. Science 307, 929-932, doi:10.1126/science.1107403 (2005).

21 Coutadeur, S. et al. A novel DYRK1A (dual specificity tyrosine phosphorylation-regulated kinase 1A) inhibitor for the treatment of Alzheimer's disease: effect on Tau and amyloid pathologies in vitro. Journal of neurochemistry 133, 440-451, doi:10.1111/jnc.13018 (2015).

22 Sengupta, A. et al. Phosphorylation of tau at both Thr 231 and Ser 262 is required for maximal inhibition of its binding to microtubules. Archives of biochemistry and biophysics 357, 299-309, doi:10.1006/abbi.1998.0813 (1998).

23 Dixit, R., Ross, J. L., Goldman, Y. E. \& Holzbaur, E. L. Differential regulation of dynein and kinesin motor proteins by tau. Science 319, 1086-1089, doi:10.1126/science.1152993 (2008).

24 Stoothoff, W. H. \& Johnson, G. V. Tau phosphorylation: physiological and pathological consequences. Biochimica et biophysica acta 1739, 280-297, doi:10.1016/j.bbadis.2004.06.017 (2005).

25 Ikezu, S. et al. Tau-tubulin kinase 1 and amyloid-beta peptide induce phosphorylation of collapsin response mediator protein-2 and enhance neurite degeneration in Alzheimer disease mouse models. Acta neuropathologica communications 8, 12, doi:10.1186/s40478-020-0890-4 (2020).

26 Gan, Z. et al. Vimentin Intermediate Filaments Template Microtubule Networks to Enhance Persistence in Cell Polarity and Directed Migration. Cell Syst 3, 252-263 e258, doi:10.1016/j.cels.2016.08.007 (2016).

27 Martin, M. \& Akhmanova, A. Coming into Focus: Mechanisms of Microtubule Minus-End Organization. Trends in cell biology 28, 574-588, doi:10.1016/j.tcb.2018.02.011 (2018).

28 Melkov, A. \& Abdu, U. Regulation of long-distance transport of mitochondria along microtubules. Cellular and molecular life sciences : CMLS 75, 163-176, doi:10.1007/s00018-017-2590-1 (2018). 
29 Lopes, S. et al. Tau Deletion Prevents Stress-Induced Dendritic Atrophy in Prefrontal Cortex: Role of Synaptic Mitochondria. Cereb Cortex 27, 2580-2591, doi:10.1093/cercor/bhw057 (2017).

30 Reddy, P. H. Abnormal tau, mitochondrial dysfunction, impaired axonal transport of mitochondria, and synaptic deprivation in Alzheimer's disease. Brain research 1415, 136-148, doi:10.1016/j.brainres.2011.07.052 (2011).

31 Ebneth, A. et al. Overexpression of tau protein inhibits kinesin-dependent trafficking of vesicles, mitochondria, and endoplasmic reticulum: implications for Alzheimer's disease. The Journal of cell biology 143, 777-794, doi:10.1083/jcb.143.3.777 (1998).

32 DuBoff, B., Gotz, J. \& Feany, M. B. Tau promotes neurodegeneration via DRP1 mislocalization in vivo. Neuron 75, 618-632, doi:10.1016/j.neuron.2012.06.026 (2012).

33 Manczak, M. \& Reddy, P. H. Abnormal interaction between the mitochondrial fission protein Drp1 and hyperphosphorylated tau in Alzheimer's disease neurons: implications for mitochondrial dysfunction and neuronal damage. Human molecular genetics 21, 2538-2547, doi:10.1093/hmg/dds072 (2012).

34 Shipley, M. M., Mangold, C. A. \& Szpara, M. L. Differentiation of the SH-SY5Y Human Neuroblastoma Cell Line. Journal of visualized experiments : JoVE, 53193, doi:10.3791/53193 (2016).

35 Frederick, R. L. \& Shaw, J. M. Moving mitochondria: establishing distribution of an essential organelle. Traffic 8, 1668-1675, doi:10.1111/j.1600-0854.2007.00644.x (2007).

36 Geiger, B., Spatz, J. P. \& Bershadsky, A. D. Environmental sensing through focal adhesions. Nature reviews. Molecular cell biology 10, 21-33, doi:10.1038/nrm2593 (2009).

37 Seetharaman, S. \& Etienne-Manneville, S. Microtubules at focal adhesions - a doubleedged sword. Journal of cell science 132, doi:10.1242/jcs.232843 (2019).

38 Ezratty, E. J., Partridge, M. A. \& Gundersen, G. G. Microtubule-induced focal adhesion disassembly is mediated by dynamin and focal adhesion kinase. Nature cell biology 7 , 581-590, doi:10.1038/ncb1262 (2005).

39 Leube, R. E., Moch, M. \& Windoffer, R. Intermediate filaments and the regulation of focal adhesion. Curr Opin Cell Biol 32, 13-20, doi:10.1016/j.ceb.2014.09.011 (2015).

40 Kilinc, D. The Emerging Role of Mechanics in Synapse Formation and Plasticity. Frontiers in cellular neuroscience 12, 483, doi:10.3389/fncel.2018.00483 (2018).

41 Robertson, L. K. \& Ostergaard, H. L. Paxillin associates with the microtubule cytoskeleton and the immunological synapse of CTL through its leucine-aspartic acid domains and contributes to microtubule organizing center reorientation. J Immunol 187, 5824-5833, doi:10.4049/jimmunol.1003690 (2011).

$42 \mathrm{Ng}$, D. H., Humphries, J. D., Byron, A., Millon-Fremillon, A. \& Humphries, M. J. Microtubule-dependent modulation of adhesion complex composition. PLoS One 9, e115213, doi:10.1371/journal.pone.0115213 (2014).

43 Sulzmaier, F. J., Jean, C. \& Schlaepfer, D. D. FAK in cancer: mechanistic findings and clinical applications. Nat Rev Cancer 14, 598-610, doi:10.1038/nrc3792 (2014).

44 Rico, B. et al. Control of axonal branching and synapse formation by focal adhesion kinase. Nature neuroscience 7, 1059-1069, doi:10.1038/nn1317 (2004). 
45 Tamura, M., Gu, J., Takino, T. \& Yamada, K. M. Tumor suppressor PTEN inhibition of cell invasion, migration, and growth: differential involvement of focal adhesion kinase and p130Cas. Cancer research 59, 442-449 (1999).

46 Trogden, K. P. et al. An image-based small-molecule screen identifies vimentin as a pharmacologically relevant target of simvastatin in cancer cells. The FASEB journal : official publication of the Federation of American Societies for Experimental Biology 32 , 2841-2854, doi:10.1096/fj.201700663R (2018).

47 Dong, J. M., Lau, L. S., Ng, Y. W., Lim, L. \& Manser, E. Paxillin nuclear-cytoplasmic localization is regulated by phosphorylation of the LD4 motif: evidence that nuclear paxillin promotes cell proliferation. The Biochemical journal 418, 173-184, doi:10.1042/BJ20080170 (2009).

48 Suresh Babu, S. et al. Mechanism of stretch-induced activation of the mechanotransducer zyxin in vascular cells. Science signaling 5, ra91, doi:10.1126/scisignal.2003173 (2012).

49 Szklarczyk, D. et al. STRING v11: protein-protein association networks with increased coverage, supporting functional discovery in genome-wide experimental datasets. Nucleic acids research 47, D607-D613, doi:10.1093/nar/gky1131 (2019).

50 Bausch-Fluck, D. et al. A mass spectrometric-derived cell surface protein atlas. PLoS One 10, e0121314, doi:10.1371/journal.pone.0121314 (2015).

51 Nunomura, K. et al. Cell surface labeling and mass spectrometry reveal diversity of cell surface markers and signaling molecules expressed in undifferentiated mouse embryonic stem cells. Mol Cell Proteomics 4, 1968-1976, doi:10.1074/mcp.M500216MCP200 (2005).

52 Smigiel, R. et al. Homozygous mutation in the Neurofascin gene affecting the glial isoform of Neurofascin causes severe neurodevelopment disorder with hypotonia, amimia and areflexia. Human molecular genetics 27, 3669-3674, doi:10.1093/hmg/ddy277 (2018).

53 Kvarnung, M. et al. Ataxia in Patients With Bi-Allelic NFASC Mutations and Absence of Full-Length NF186. Frontiers in genetics 10, 896, doi:10.3389/fgene.2019.00896 (2019).

54 Davis, T. A., Loos, B. \& Engelbrecht, A. M. Corrigendum to AHNAK: The giant jack of all trades [Cell. Signal. 26 (2014) 2683-2693]. Cellular signalling 27, 187-188, doi:10.1016/j.cellsig.2014.10.004 (2015).

55 Moreno-Layseca, P., Icha, J., Hamidi, H. \& Ivaska, J. Integrin trafficking in cells and tissues. Nature cell biology 21, 122-132, doi:10.1038/s41556-018-0223-z (2019).

56 Bakker, J., Spits, M., Neefjes, J. \& Berlin, I. The EGFR odyssey - from activation to destruction in space and time. Journal of cell science 130, 4087-4096, doi:10.1242/jcs.209197 (2017).

57 Galea, G. \& Simpson, J. C. High-content analysis of Rab protein function at the ERGolgi interface. Bioarchitecture 5, 44-53, doi:10.1080/19490992.2015.1102826 (2015).

58 Naguib, A. et al. PTEN functions by recruitment to cytoplasmic vesicles. Mol Cell 58, 255-268, doi:10.1016/j.molcel.2015.03.011 (2015).

59 Kii, I., Nishiyama, T. \& Kudo, A. Periostin promotes secretion of fibronectin from the endoplasmic reticulum. Biochemical and biophysical research communications 470, 888893, doi:10.1016/j.bbrc.2016.01.139 (2016). 
60 Noordstra, I. \& Akhmanova, A. Linking cortical microtubule attachment and exocytosis. F1000Res 6, 469, doi:10.12688/f1000research.10729.1 (2017).

61 Pollerberg, G. E., Thelen, K., Theiss, M. O. \& Hochlehnert, B. C. The role of cell adhesion molecules for navigating axons: density matters. Mechanisms of development 130, 359-372, doi:10.1016/j.mod.2012.11.002 (2013).

62 Takano, T. et al. Chemico-genetic discovery of astrocytic control of inhibition in vivo. Nature 588, 296-302, doi:10.1038/s41586-020-2926-0 (2020).

63 Bhat, M. A. et al. Axon-glia interactions and the domain organization of myelinated axons requires neurexin IV/Caspr/Paranodin. Neuron 30, 369-383, doi:10.1016/s08966273(01)00294-x (2001).

64 Zonta, B. et al. A critical role for Neurofascin in regulating action potential initiation through maintenance of the axon initial segment. Neuron 69, 945-956, doi:10.1016/j.neuron.2011.02.021 (2011).

65 Sistani, L. et al. Neuronal proteins are novel components of podocyte major processes and their expression in glomerular crescents supports their role in crescent formation. Kidney international 83, 63-71, doi:10.1038/ki.2012.321 (2013).

66 Valiente, M. et al. Binding of PTEN to specific PDZ domains contributes to PTEN protein stability and phosphorylation by microtubule-associated serine/threonine kinases. The Journal of biological chemistry 280, 28936-28943, doi:10.1074/jbc.M504761200 (2005).

67 Pan, C. et al. Hsp90B enhances MAST1-mediated cisplatin resistance by protecting MAST1 from proteosomal degradation. The Journal of clinical investigation 129, 41104123, doi:10.1172/JCI125963 (2019).

68 McClellan, A. J. et al. Diverse cellular functions of the Hsp90 molecular chaperone uncovered using systems approaches. Cell 131, 121-135, doi:10.1016/j.cell.2007.07.036 (2007).

69 Xiong, X. et al. Heat shock protein 90beta stabilizes focal adhesion kinase and enhances cell migration and invasion in breast cancer cells. Experimental cell research 326, 78-89, doi:10.1016/j.yexcr.2014.05.018 (2014).

70 Quinta, H. R. et al. Management of cytoskeleton architecture by molecular chaperones and immunophilins. Cellular signalling 23, 1907-1920, doi:10.1016/j.cellsig.2011.07.023 (2011).

71 Wu, Y., Ding, Y., Zheng, X. \& Liao, K. The molecular chaperone Hsp90 maintains Golgi organization and vesicular trafficking by regulating microtubule stability. Journal of molecular cell biology 12, 448-461, doi:10.1093/jmcb/mjz093 (2020).

72 Chen, C. Y. \& Balch, W. E. The Hsp90 chaperone complex regulates GDI-dependent Rab recycling. Molecular biology of the cell 17, 3494-3507, doi:10.1091/mbc.e05-121096 (2006).

73 Shinde, S. R. \& Maddika, S. PTEN modulates EGFR late endocytic trafficking and degradation by dephosphorylating Rab7. Nature communications 7, 10689, doi:10.1038/ncomms10689 (2016).

74 Genest, O., Wickner, S. \& Doyle, S. M. Hsp90 and Hsp70 chaperones: Collaborators in protein remodeling. The Journal of biological chemistry 294, 2109-2120, doi:10.1074/jbc.REV118.002806 (2019). 
75 Parfitt, D. A. et al. The ataxia protein sacsin is a functional co-chaperone that protects against polyglutamine-expanded ataxin-1. Human molecular genetics 18, 1556-1565, doi:10.1093/hmg/ddp067 (2009).

76 Gavriilidis, C. et al. The MTM1-UBQLN2-HSP complex mediates degradation of misfolded intermediate filaments in skeletal muscle. Nature cell biology 20, 198-210, doi:10.1038/s41556-017-0024-9 (2018).

77 Giustiniani, J. et al. Tubulin acetylation favors Hsp90 recruitment to microtubules and stimulates the signaling function of the Hsp90 clients Akt/PKB and p53. Cellular signalling 21, 529-539, doi:10.1016/j.cellsig.2008.12.004 (2009).

78 Lackie, R. E. et al. The Hsp70/Hsp90 Chaperone Machinery in Neurodegenerative Diseases. Frontiers in neuroscience 11, 254, doi:10.3389/fnins.2017.00254 (2017).

79 Berglund, E. O. et al. Ataxia and abnormal cerebellar microorganization in mice with ablated contactin gene expression. Neuron 24, 739-750, doi:10.1016/s08966273(00)81126-5 (1999).

80 Sakurai, T. et al. Overlapping functions of the cell adhesion molecules Nr-CAM and L1 in cerebellar granule cell development. The Journal of cell biology 154, 1259-1273, doi:10.1083/jcb.200104122 (2001).

81 Lariviere, R. et al. Sacs R272C missense homozygous mice develop an ataxia phenotype. Molecular brain 12, 19, doi:10.1186/s13041-019-0438-3 (2019).

82 Xing, L. et al. Layer specific and general requirements for ERK/MAPK signaling in the developing neocortex. eLife 5, doi:10.7554/eLife.11123 (2016). 
bioRxiv preprint doi: https://doi.org/10.1101/2021.08.20.456807; this version posted September 2, 2021. The copyright holder for this preprint (which was not certified by peer review) is the author/funder, who has granted bioRxiv a license to display the preprint in perpetuity. It is made available under aCC-BY-NC-ND 4.0 International license.

Fig. 1 a

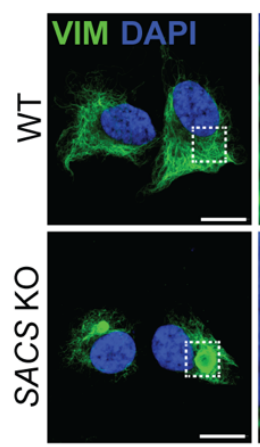

g

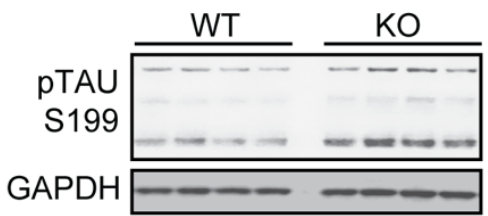

h

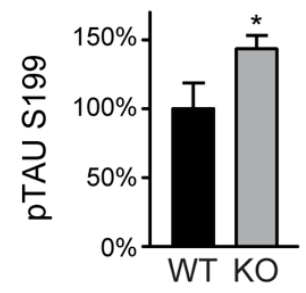

i

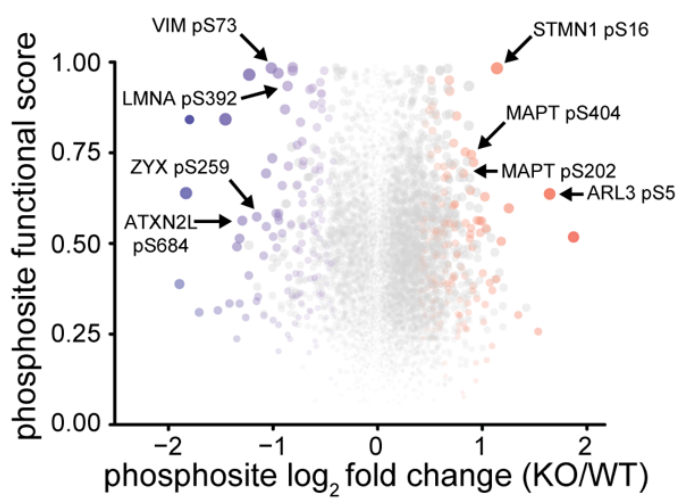

C

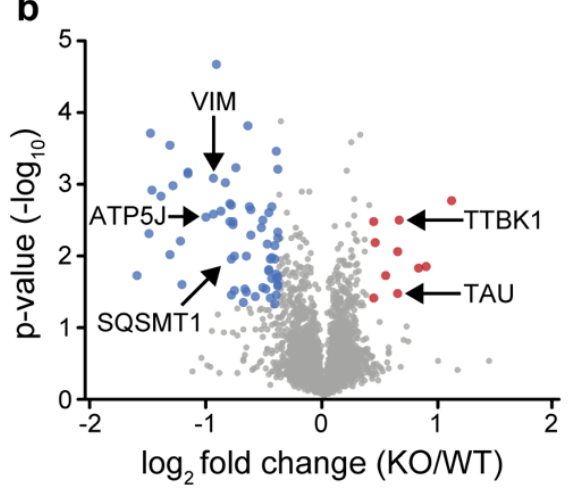

j

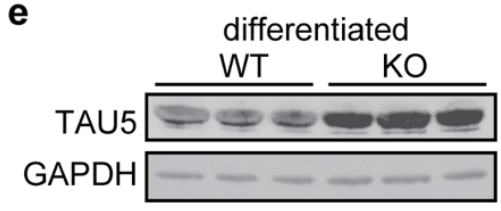

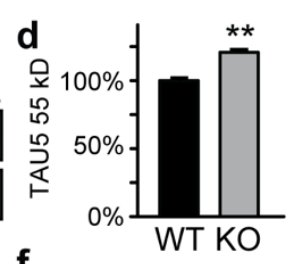

f

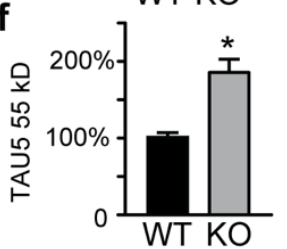

k

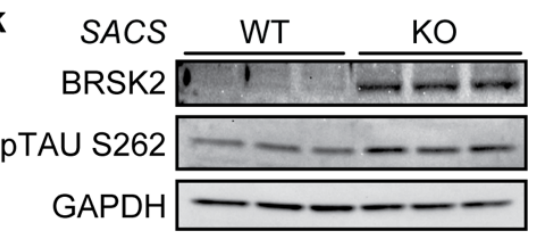

GAPDH
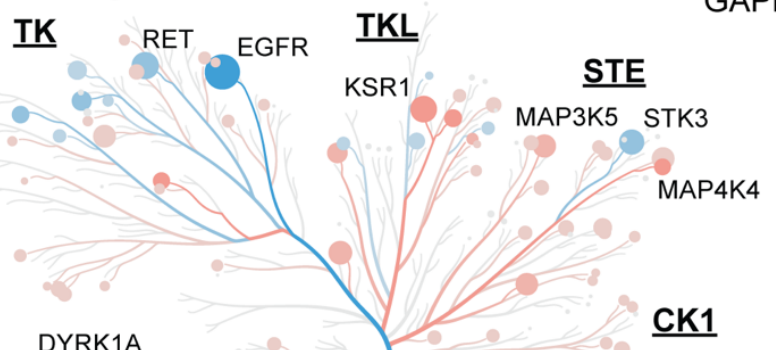

CMGC
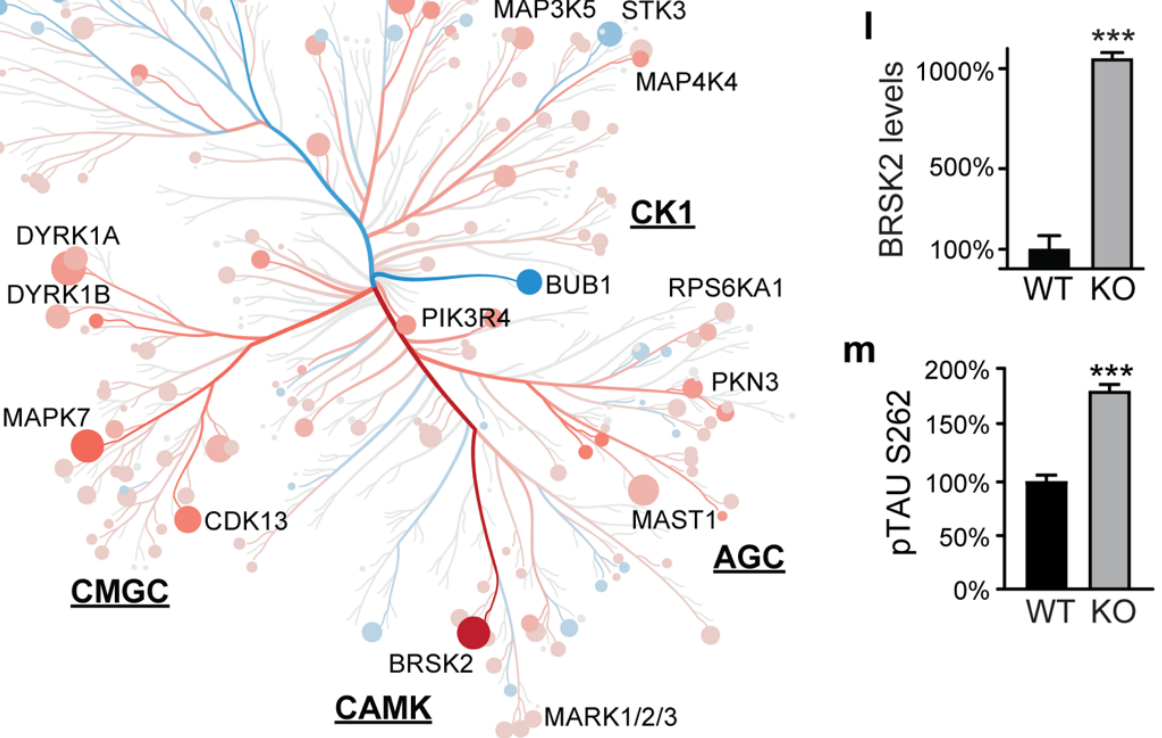

m

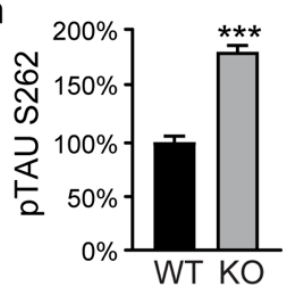

n

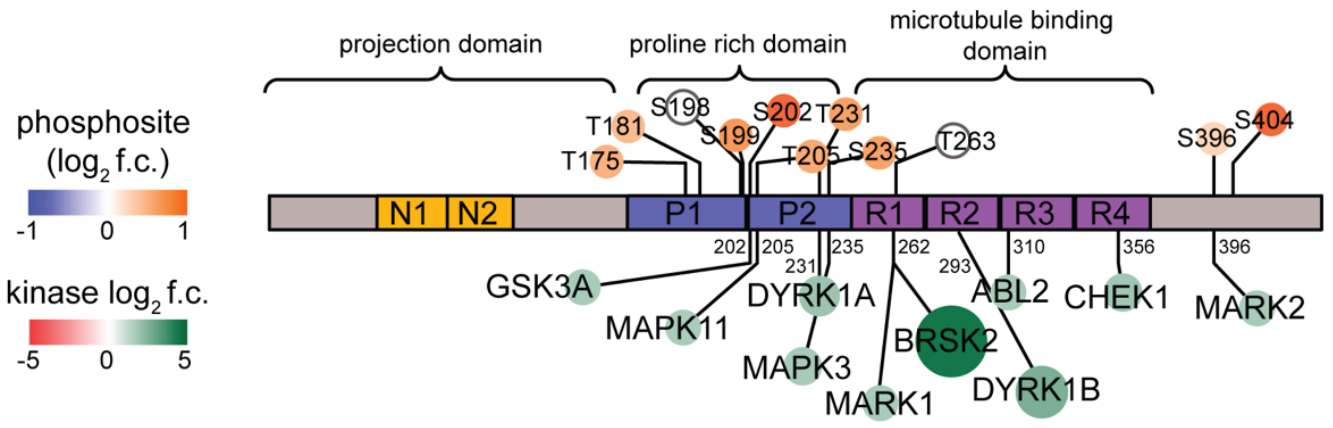

\section{Figure 1 - Proteomic profiling of SACS KO cells}

a. Representative confocal images of control (WT) and SACS knockout (KO) SH-SY5Y neuroblastoma cells immunostained for the intermediate filament protein vimentin. Scale bar $=10 \mu \mathrm{m}$.

b. Global proteomic profiling of SACS KO SH-SY5Y cells (SACS KO). Cutoffs for significance were $p<0.05$ and $\log _{2}$ fold change $-/+0.4$. 
c-f. Western Blot analysis quantification of pan-tau (Tau5) in SACS KO and WT cells in undifferentiated $(c, d)$ and neuronally differentiated (e,f) SH-SY5Y cells.

$\mathrm{g}, \mathrm{h}$. Western blot and quantification of phosphorylated tau at serine 199.Phosphoproteomic analysis of SACS KO SH-SY5Y cells. Green circles mark specific phosphorylated residues on tau.

i. Functional analysis of altered phosphosites in SACS KO cells. Y-axis is the functional score assigned by Ochoa et al (2020), which combines 59 features to assess the imact of each phosphosite on organismal fitness. Dot color and size reflects a combined metric of $\log _{2}$ fold change and functional score (product of the two together). Non-significant phosphosites are colored grey.

j. Phylogenetic tree of the kinome in SACS KO cells. $35 \%$ of kinases were altered in SACS KO cells ( $\log _{2}$ f.c. $-/+0.4, p<0.05)$. Color indicates $\log _{2}$ f.c. of kinase abundance, size indicates $-\log _{10} p$-value. Underlined abbreviations refer to phylogenetically related kinase families.

k-m. Western blot and quantification for BRSK2, and the BRSK2 target residue pTAU Ser262.

n. Protein map of tau isoform 2 (2N4R). Phosphosites identified in phosphoproteomic profiling are labelled above diagram. Tau kinases identified in the kinome profiling are listed below, labeled with known amino acid substrates in tau. Colored circles correlate with log2FC of significantly differentially expressed phosphosites or kinases.

Unless otherwise noted, all error bars are S.E.M., all statistical tests are Student's t-test $\left({ }^{*} \mathrm{P}<0.05,{ }^{* *} \mathrm{P}<0.01\right.$, $\left.{ }^{* * *} \mathrm{P}<0.001,{ }^{* * * *} \mathrm{P}<0.0001\right)$. 


\section{Fig. 2}

a
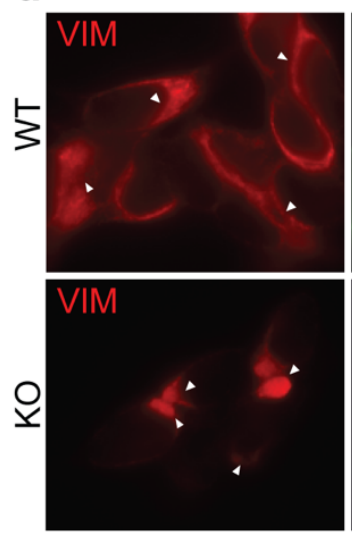

d

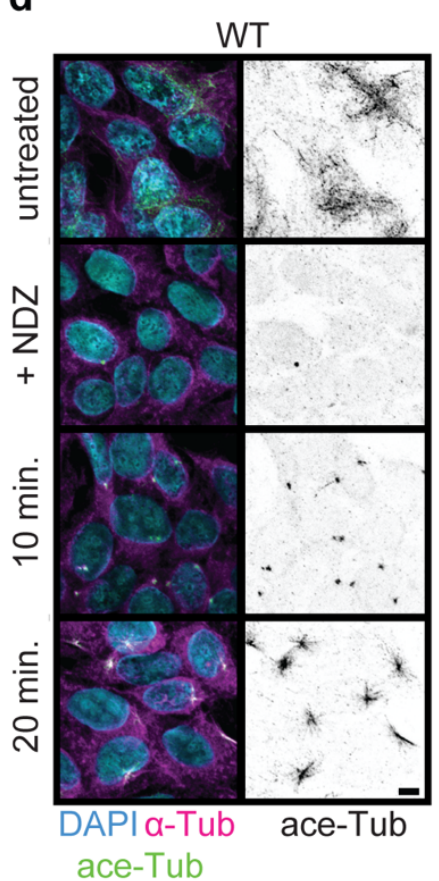

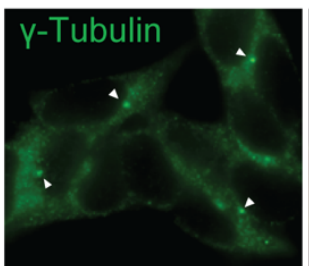
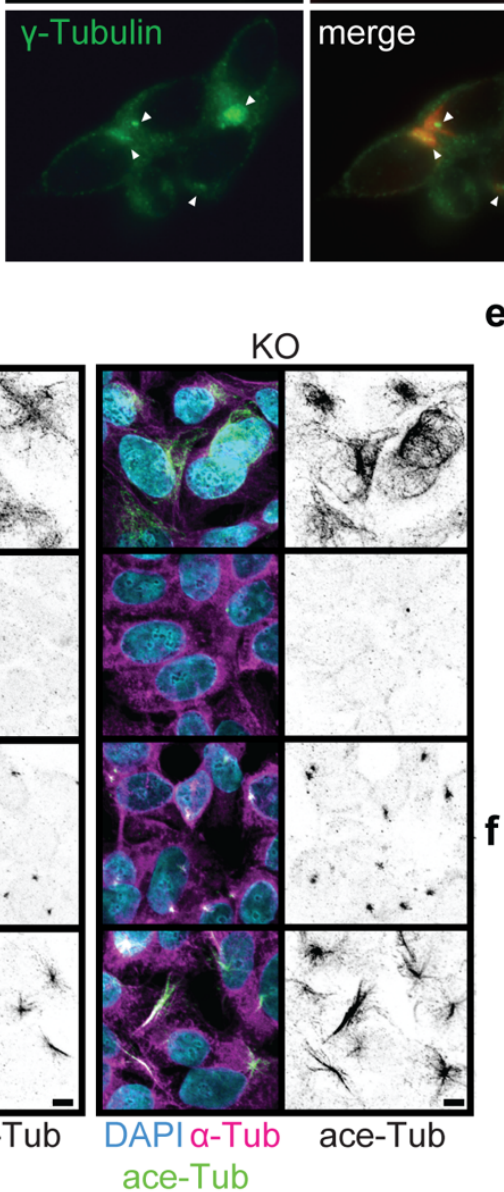

b
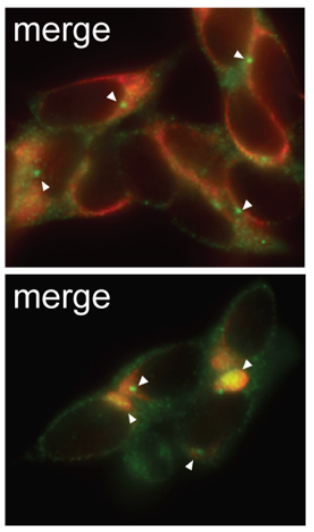
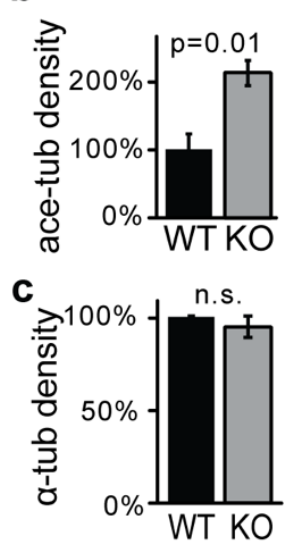

e -- WT aTub - WT aceTub

- KO aTub - KO aceTub

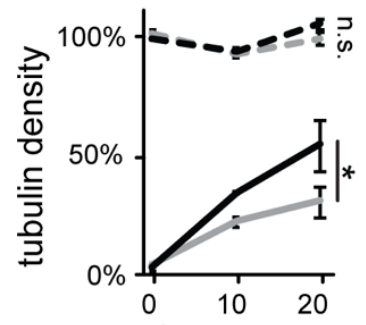

min. after washout

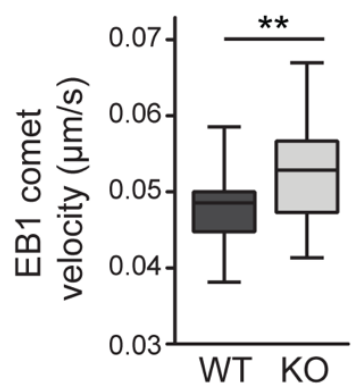

\section{Figure 2 - Altered microtubule structure and dynamics in SACS KO cells}

a. Confocal immunofluorescent images of SACS WT/KO cells stained for vimentin, and the MTOC marker gamma tubulin. Arrowheads point to the most intense signal in each cell, showing that vimentin bundles surround the MTOC in SACS KO cells. Scale bar $=10 \mu \mathrm{m}$.

b-c. Quantification of images in Extended Data Fig. 2b, showing altered microtubule acetylation in SACS KO cells (b) without global changes in microtubule structure (c).

d. Confocal images of WT/KO cells treated with nocodazole (NDZ) labeled for alpha- and acetylated-tubulin at indicated time points following nocodazole washout. Note the faster microtubule repolymerization and acetylation in SACS KO cells. Scale bar $=10 \mu \mathrm{m}$.

e. Quantification of images in (d). $n=3$ coverslips; One-way ANOVA with Tukey post-test.

f. Quantification of microtubule polymerization velocity marked by EB1-GFP movement in WT/KO cells on TIRF microscope from Supplemental Video 1. $n=34$ WT and $n=25$ SACS KO cells, examined from at least three independent experiments; unpaired t-test. 
bioRxiv preprint doi: https://doi.org/10.1101/2021.08.20.456807; this version posted September 2, 2021. The copyright holder for this preprint

(which was not certified by peer review) is the author/funder, who has granted bioRxiv a license to display the preprint in perpetuity. It is made available under aCC-BY-NC-ND 4.0 International license.

\section{Fig. 3}

a

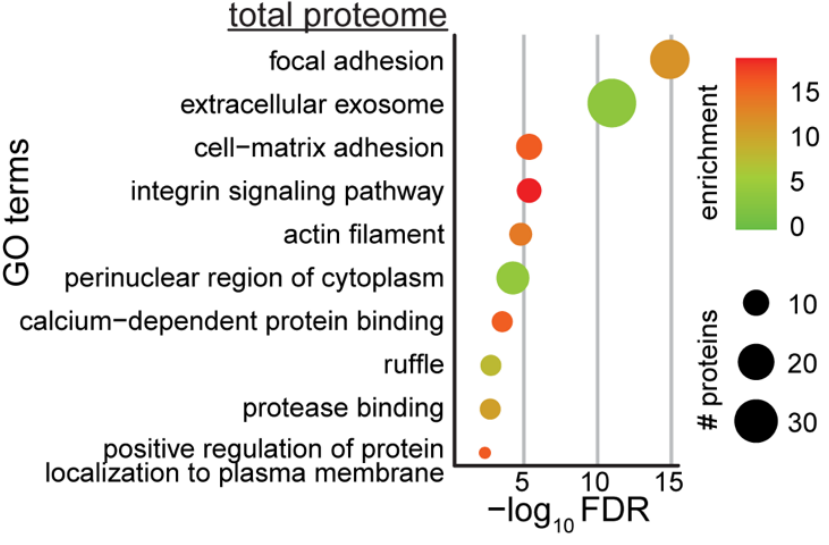

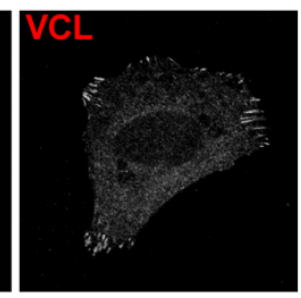

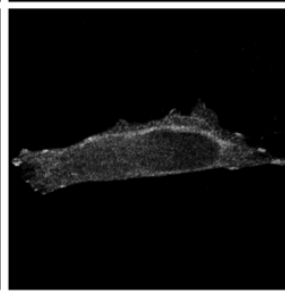

merge

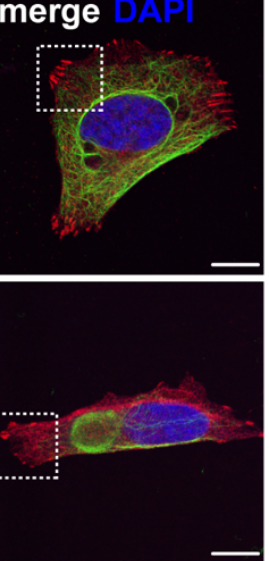

b hypophosphorylated proteins

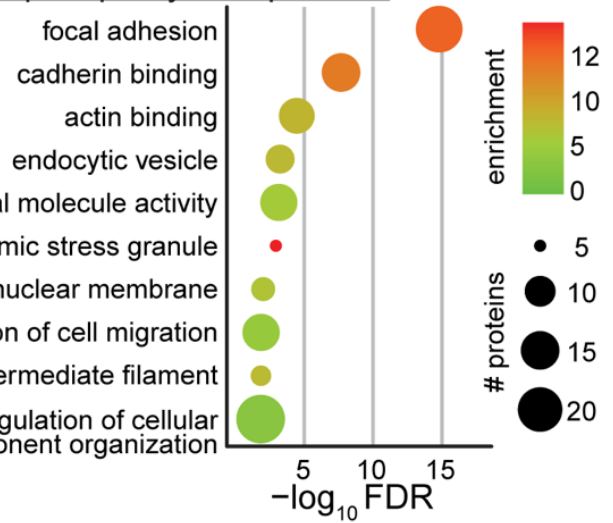

structural molecule activity

cytoplasmic stress granule

nuclear membrane

regulation of cell migration

intermediate filament

regulation of cellular component organization

d

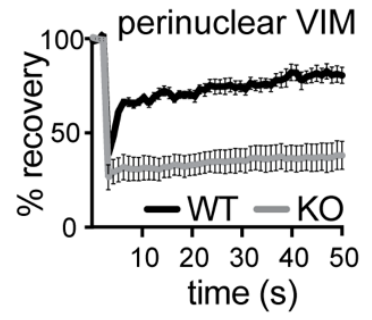

e

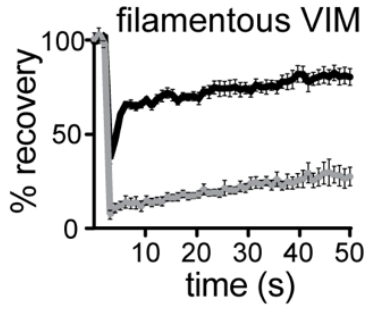

f

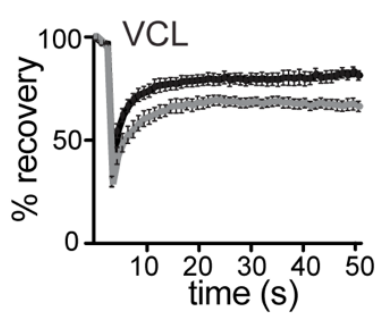

g

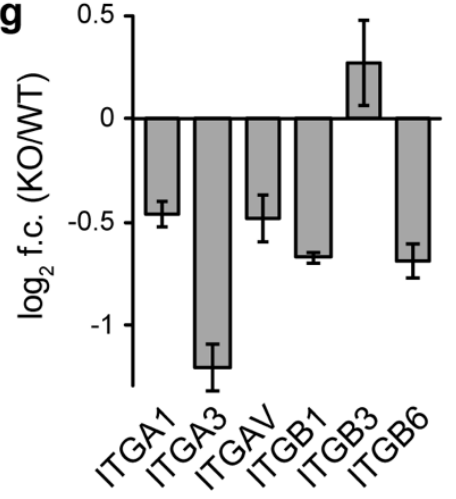

h VIM

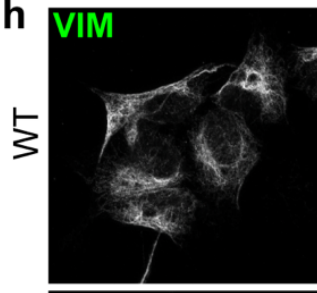

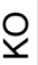
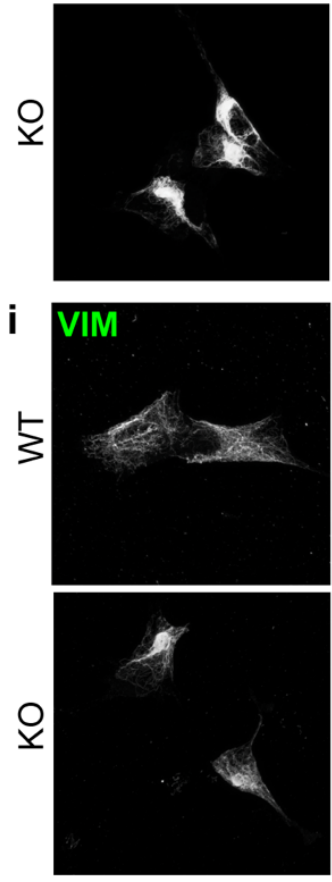
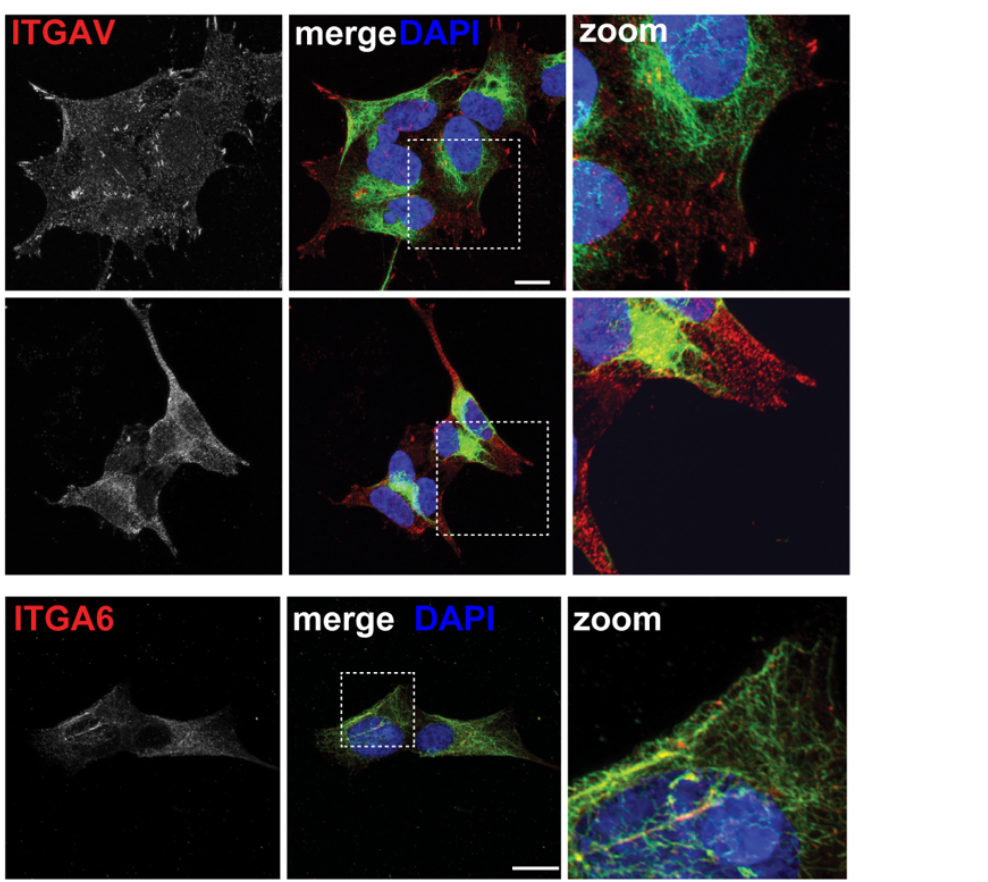

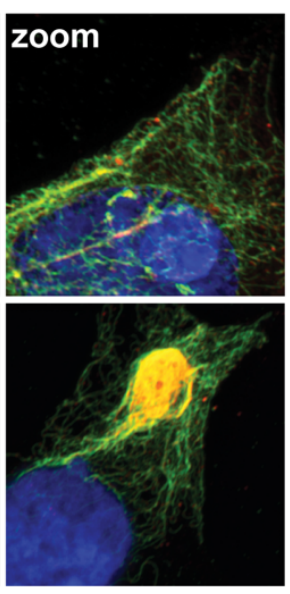




\section{Figure 3 - Focal Adhesions are altered in SACS KO cells}

a,b.GO term analysis from the total proteome $\left(p<0.05, \log _{2}\right.$ fold change cutoff $\left.-/+0.4\right)(a)$, and hypophosphorylated $\left(p<0.05, \log _{2}\right.$ fold change $\left.<-0.4\right)($ (b) proteins. Both identify focal adhesions as the main process that is altered in SACS KO cells.

c. Confocal images of WT/KO SH-SY5Y cells immunolabeled for vimentin and the focal adhesion protein vinculin.

d-f. FRAP analysis of perinuclear vimentin (d), filamentous vimentin on the periphery of the cell away from vimentin bundle $(e)$, and the focal adhesion protein vinculin (f). Cells were transfected with tomatoVIM/VCL expression vector and defined $2 \times 2 \mu \mathrm{m}$ regions of interest were bleached by using a 568-nm laser line. Recovery was monitored over 50 cycles of imaging with a 1-s interval. $n=10$ cells from each of three independent experiments.

g. Changes in levels of integrin proteins quantified by mass-spectrometry (Fig. 1b).

h,i. Representative confocal images of cells immunolabeled for ITGAV (h) and ITGA6 (i). Scale bar $=10 \mu \mathrm{m}$. 


\section{Fig. 4}

a

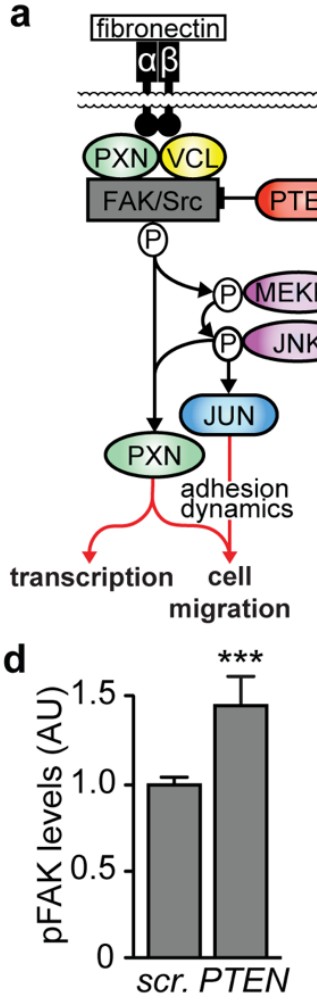

b

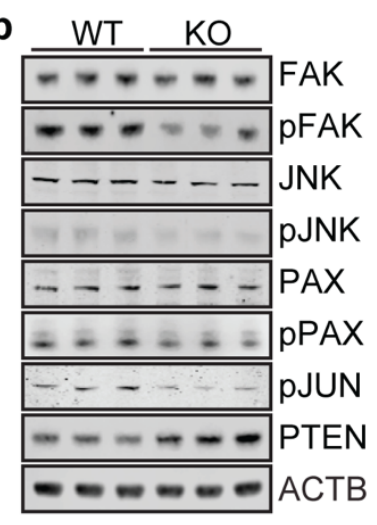

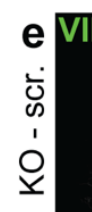

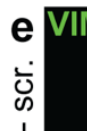

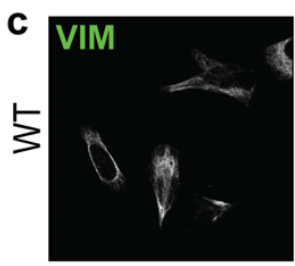
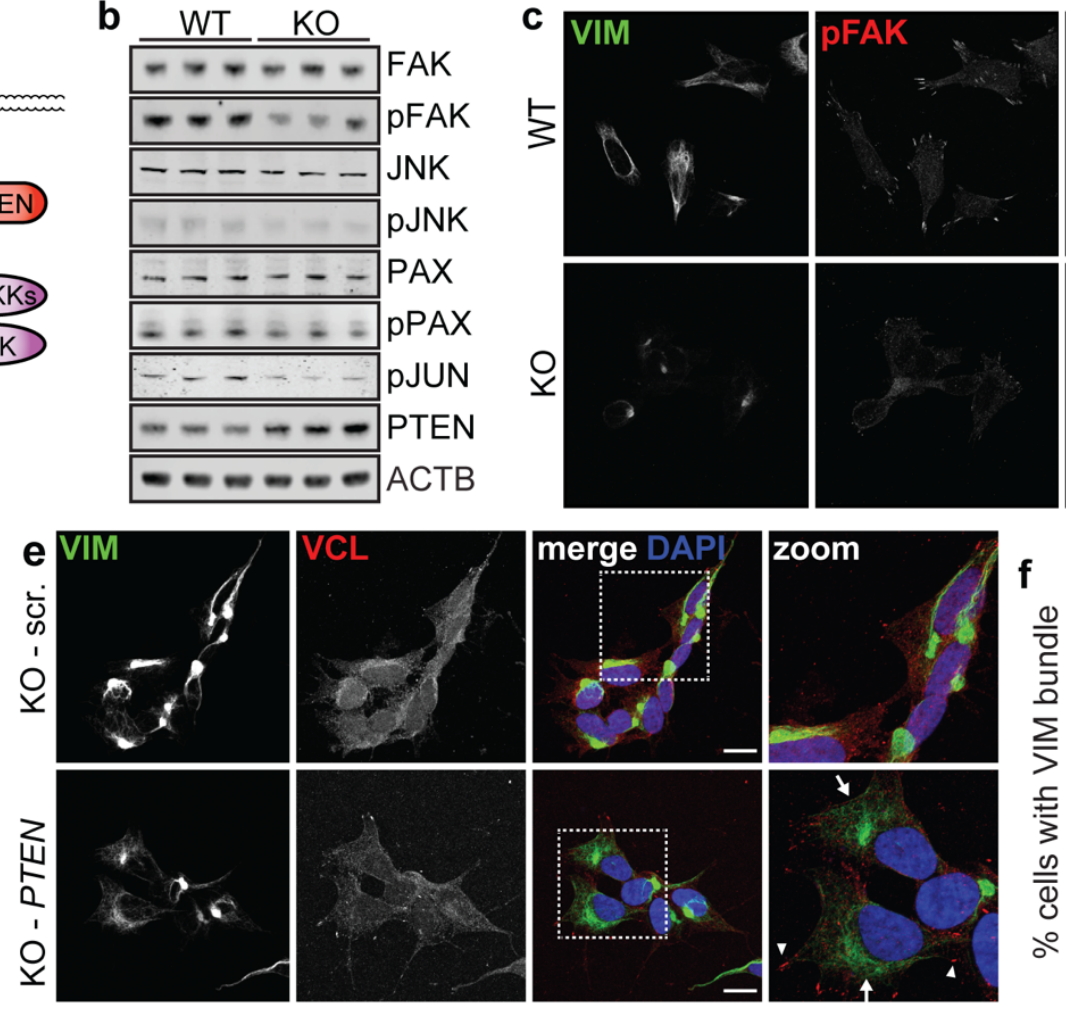

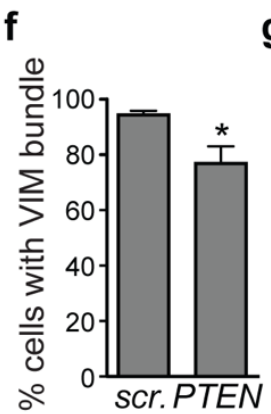

g
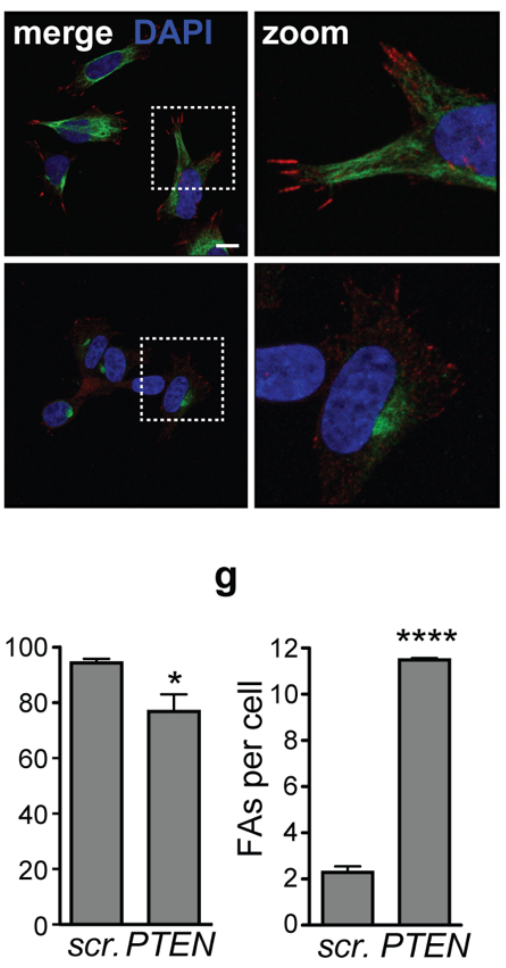

Figure 4 - Targeting upstream focal adhesion regulator PTEN rescues focal adhesion and vimentin bundling phenotypes in SACS KO cells.

a. Model of regulators and effectors of focal adhesion signaling

b. Western blots for regulators PTEN, FAK, phosphorylated FAK (pFAK), JNK, phosphorylated JNK (pJNK), paxillin (PAX), phosphorylated paxillin ( $p P A X)$ and phosphorylated Jun ( $p J U N)$ in total cell lysates from SACS KO and control cells. $\beta$-actin used to confirm equivalent sample loading.

c. Representative confocal images of cells immunolabeled for pFAK. Scale bar $=10 \mu \mathrm{m}$.

d. pFAK levels with SACS KO cells treated with either scrambled (scr.) or siRNA targeting PTEN.

e. Representative confocal images for cells transfected with siRNAs targeting PTEN (PTEN) or scramble siRNAs and immunolabeled for vimentin and vinculin. Arrowheads in the zoomed panel indicate cells with prominent focal adhesions, arrows indicate cells with absent or reduced perinuclear accumulations of vimentin. Scale bars $=10 \mu \mathrm{m}$.

$f, g$. Quantification of the incidence of sacsin KO cells with perinuclear accumulations of vimentin (f) or vinculin positive focal adhesions (g) 48 hours after transfection with siRNAs targeting PTEN or scr. siRNAs. $\mathrm{n}=3$ replicates with $>100$ cells in each replicate. 


\section{Fig. 5}

a

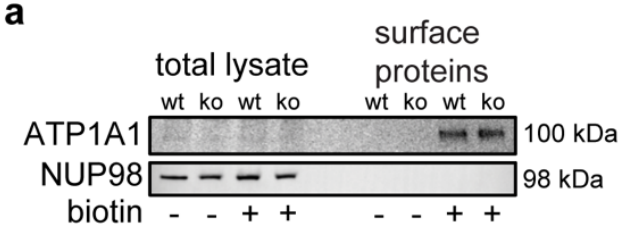

b

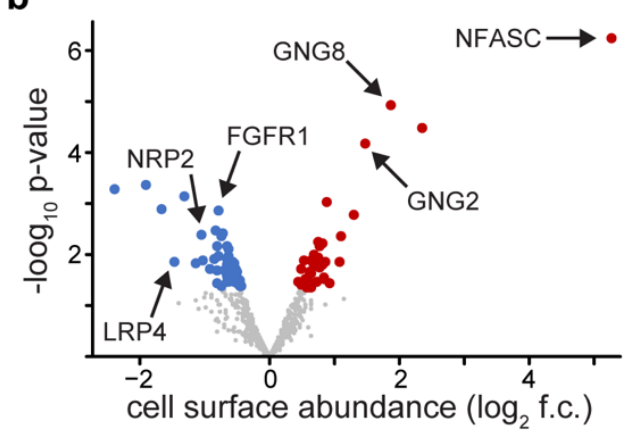

C

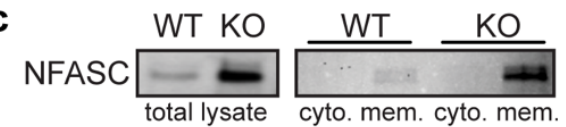

d

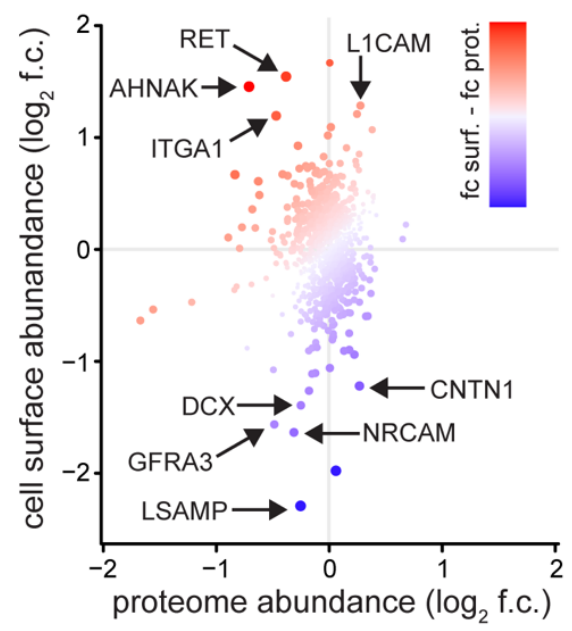

e

cell surface proteome extracellular exosome Golgi membrane GDP binding ER membrane phagocytic vesicle membrane secretory granule membrane axonogenesis neuronal cell body lysosomal membrane synapse organization ${ }^{2}-\log 10^{4}$ (FDR)

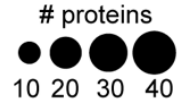

enrichment $\begin{array}{lll}0 & 5 & 10\end{array}$ f

g
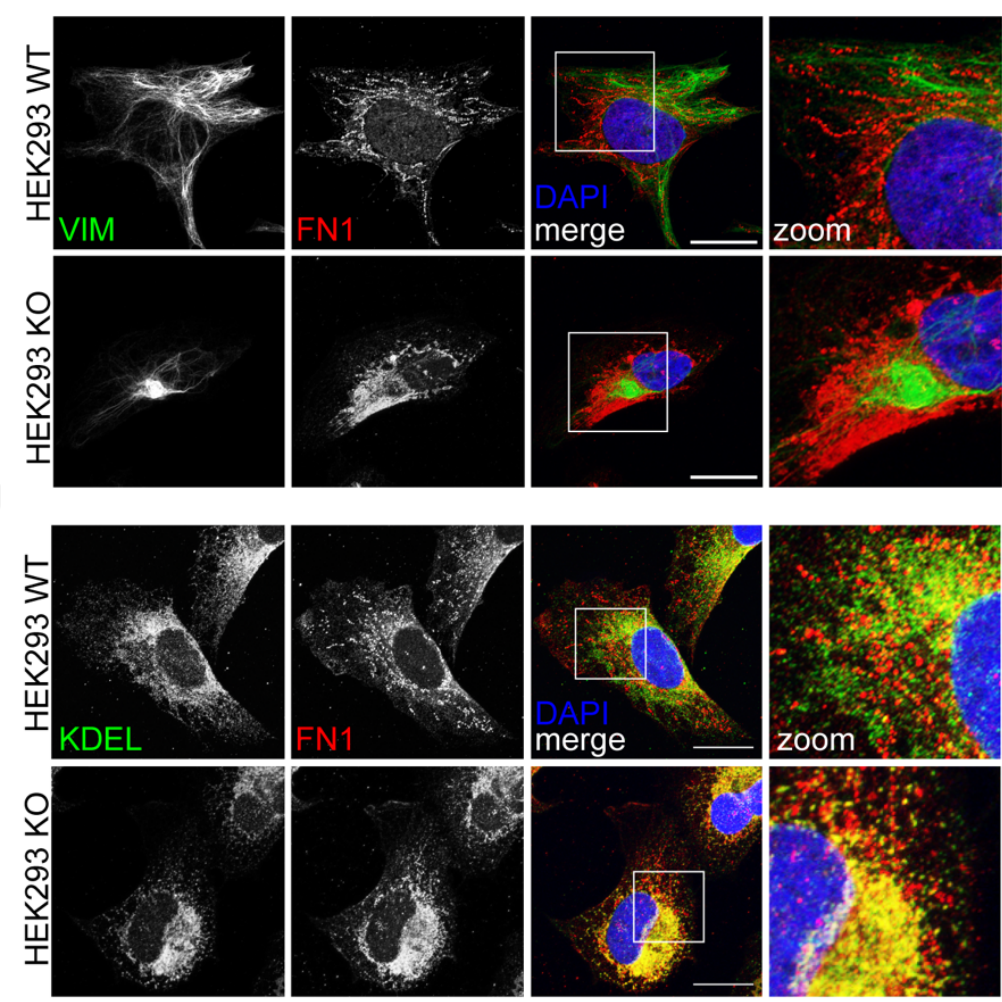

h

\section{$\underline{\text { IPA disease term }}$}

movement disorders

familial encephalopathy

abnormal morph. of nerv. system

neurodegeneration

neuromuscular disease

progressive neurological disorder

autosomal recessive neuro. disorder

tremor

disorder of basal ganglia

abnormal morphology of neurons

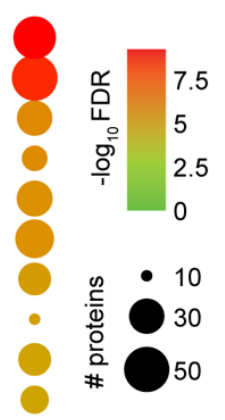

Figure 5 - Cell surface proteomics suggests deficits in neuronal adhesion molecules is a causal molecular deficit in ARSACS

a. Western blot of membrane purification approach, illustrated by ATP1A1, a membrane bound $\mathrm{Na} / \mathrm{K}$ ATPase, and NUP98, a nuclear pore protein. In total lysate only NUP98 is detectable. After purification ATP1A1 is detectable only in conditions that were treated with biotin, and NUP98 is not longer detected, suggested labelling specificity and enrichment of cell surface proteins.

b. Volcano plot of cell surface proteins only detected in surface proteomic experiment.

c. Western blot of NFASC in total lysate (left), and fractionated cytoplasmic or membrane fractions in WT and SACS KO cells. 
d. Levels of proteins detected in both cell surface and proteomic datasets. Proteins are colored by the disparity between these two datasets (fold change surface - fold chance proteome), with red indicating more, and blue less membrane abundance relative to total protein levels.

e. GO term analysis of proteins differentially localized in membrane of $S A C S$ KO cells $\left(p<0.05, \log _{2}\right.$ fold change -/+ 0.4).

f,g. Representative confocal images for fibronectin (levels not affected in any proteomic experiment) and vimentin (f) and ER marker KDEL (g) in WT and SACS KO HEK293 cells. Scale bar $=10 \mu \mathrm{m}$.

h. Disease enrichment analysis with Ingenuity Pathway Analysis (IPA) of significantly differentially expressed cell-surface proteins $\left(p<0.05, \log _{2}\right.$ fold change $\left.-/+0.4\right)$. 


\section{Fig. 6}

a
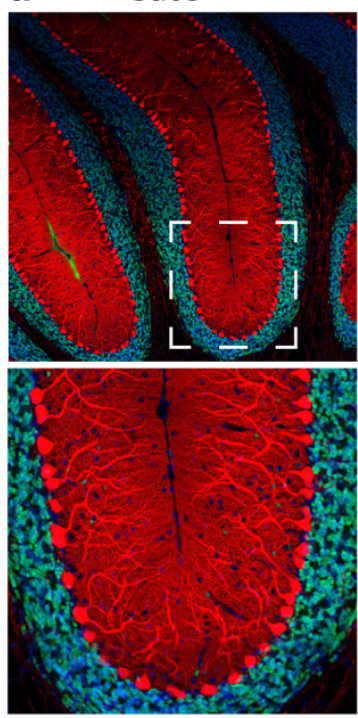

CALB1 NEUN DAPI
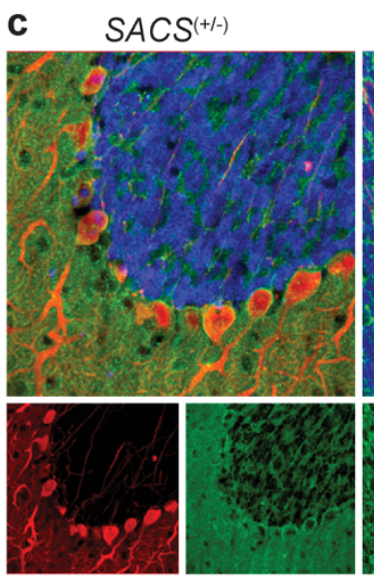

CALB1 NFASC NEUN

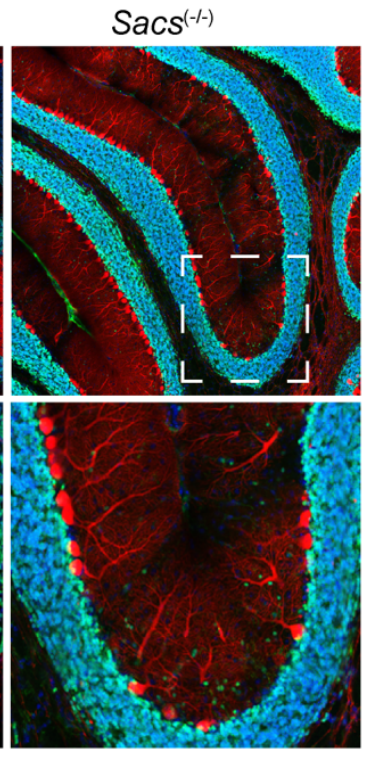

$\operatorname{SACS}^{(--)}$
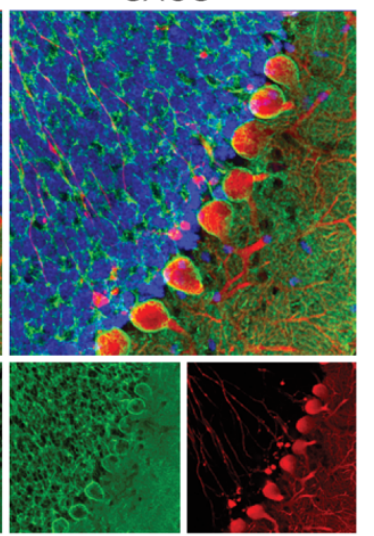

b $S A C S^{(++)}$
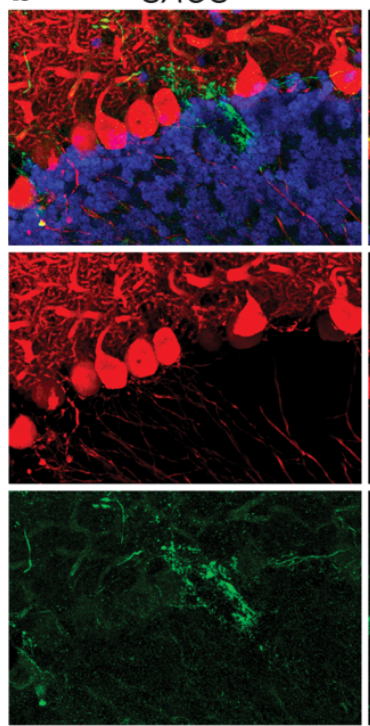

ITGA1 CALB1 NEUN

CALB1 NEUN DAPI
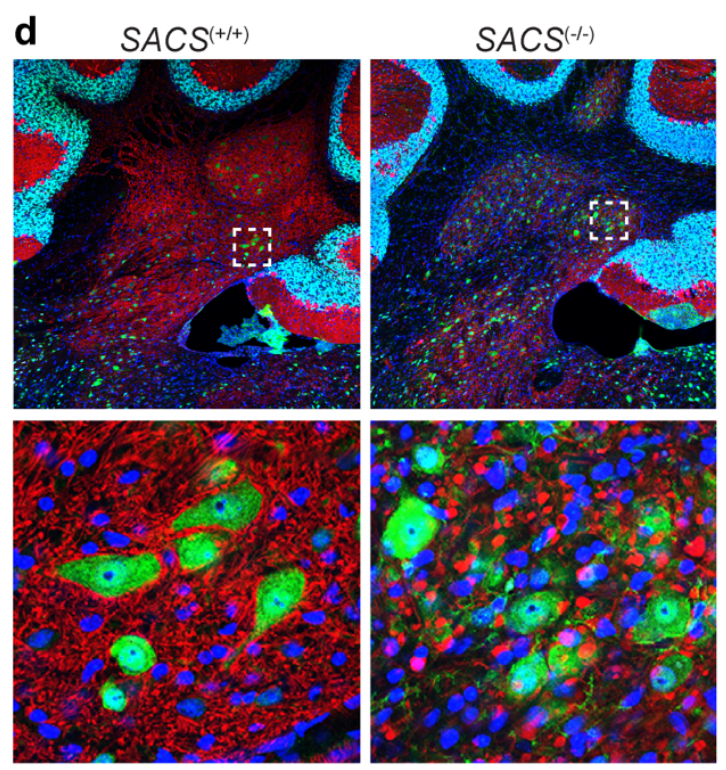

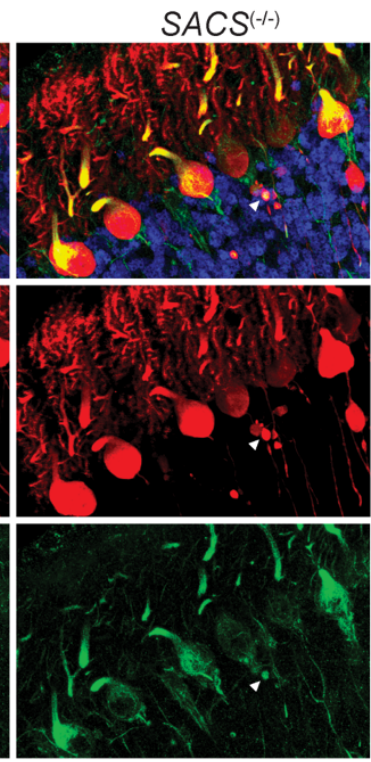

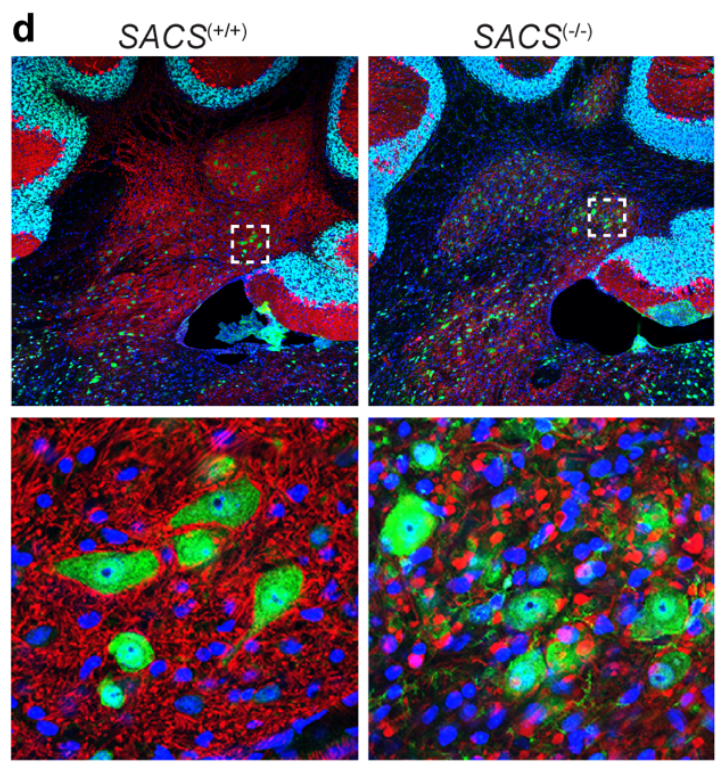

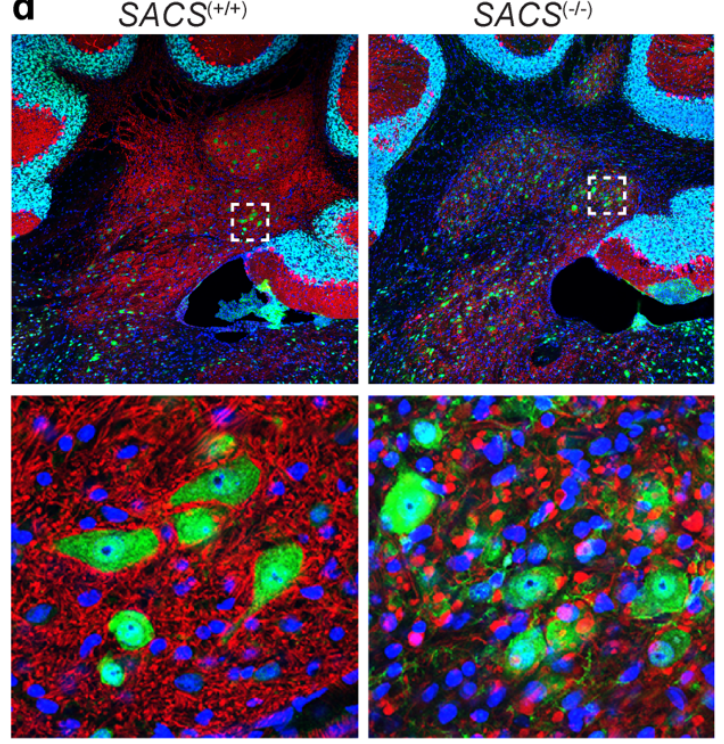

CALBINEUN DAPI

\section{Figure 6 - Altered localization of membrane proteins and synapses in ARSACS mice}

a. Confocal imaging of Purkinje neurons in litter mate controlled P120 $S A C S^{(+-)}$and $S A C S^{(--)}$mice. Purkinje marker calbindin (CALB1), neuronal marker (NEUN), and nuclei (DAPI). $S A C S^{(+-)}$mice are phenotypically normal, analogous to unaffected human carriers.

b. High magnification of Purkinje neuron layer in P120 mice, stained for integrin A1 (ITGA1), one of the most significantly mislocalized proteins in mass-spec (Fig. 6b,c). ITGA1, normally localized along axon tracts (Extended Data Fig. 6a), accumulated in initial dendritic segment in SACS KO mice. Arrowhead marks ITGA1 accumulation in axonal swellings, typical of ARSACS mice.

c. High magnification of Purkinje neuron layer in P120 mice, stained for neurofascin (NFASC). NFASC is normally localized to the axon initial segment (AIS). In SACS KO mice NFASC staining is more intense around Purkinje soma, and more prominent around the entire soma, as opposed to primarily adjacent to the AIS.

d. Confocal imaging of the DCN in P120 SACS KO mice, demonstrating synaptic deficits between Purkinje neurons and their synaptic termini on DCN neurons. 
Fig. 7
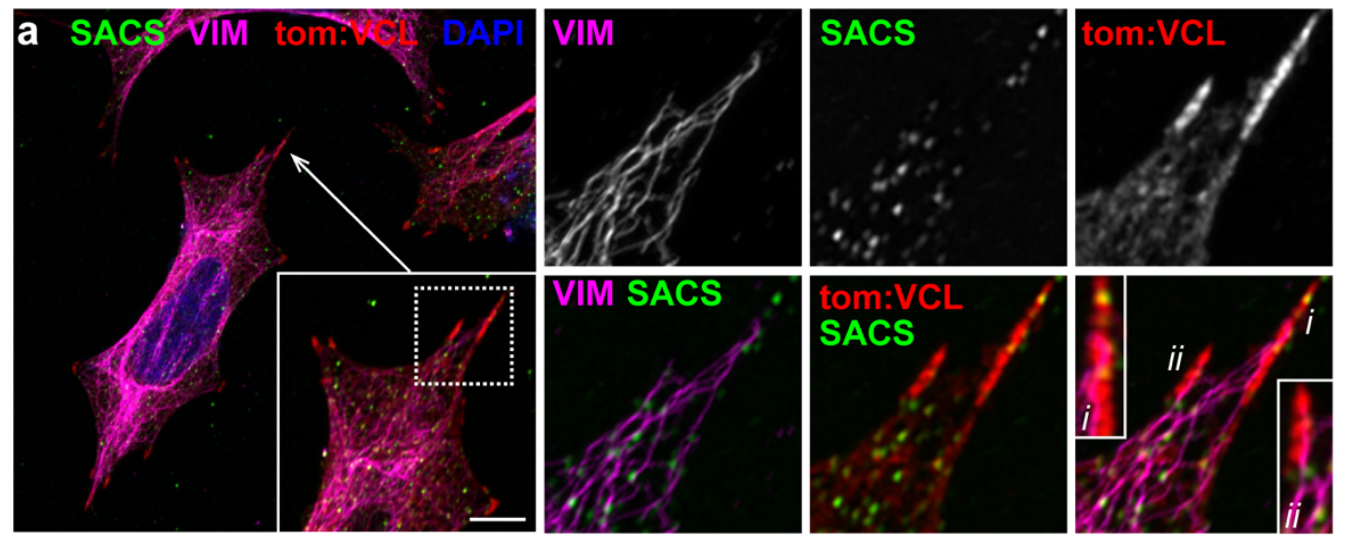

b

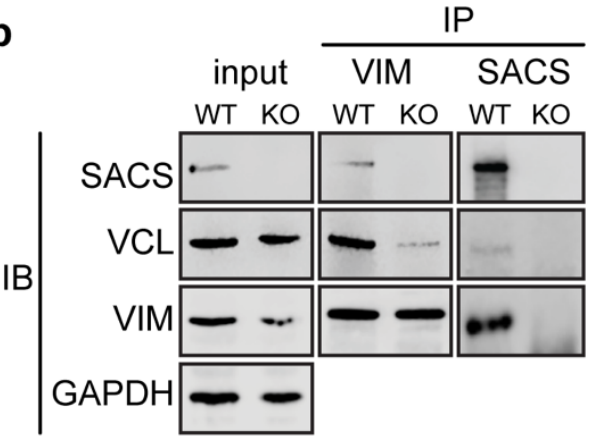

d

cluster 1
C
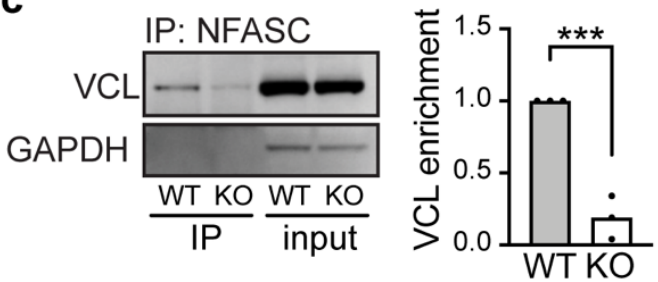

\section{cluster 2}

chaperones, microtubules, \& NCAMs

intermediate filaments

$\&$ focal adhesions

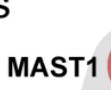

$\underline{\text { SACS interaction }}$

proteomic dataset prote-
ome surface-
ome

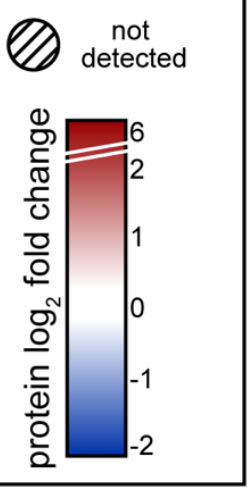

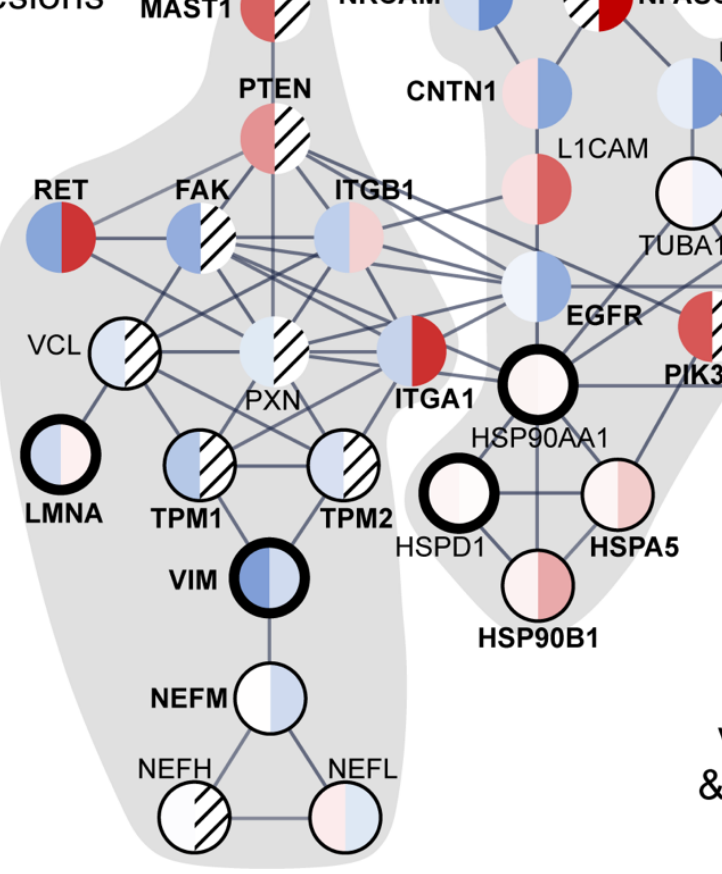

\section{DYRK1A/B}

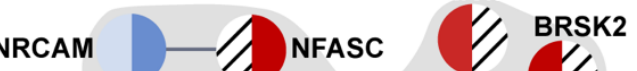

DCX

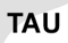

$1 /$

TAU

UBA4A

RAB7A

RAB21

RAB8A

RAB10

RAB2A

cluster 3

vesicular trafficking

\& membrane docking 


\section{Figure 7 - The loss of Sacsin disrupts protein-protein interactions}

a. Representative Airyscan confocal analysis of sacsin, vimentin, and transfected tdTomato:vinculin staining in WT SH-SY5Y cells, demonstrating SACS localization along vimentin tracts and focal adhesions. Scale bar $=10 \mu \mathrm{m}$.

b. Vimentin or sacsin were immunoprecipitated from WT and SACS KO SH-SY5Y cells, and coimmunoprecipitated proteins (sacsin, vinculin, vimentin) were analyzed by Western blot. Results suggest decreased interaction between vimentin and vinculin in SACS KO cells.

c. Co-IP of NFASC and vinculin in WT and SACS KO cells. Vinculin was not detected in secondary antibody only control blots (not shown), suggesting a specific interaction. Quantification of $n=3$ co-IP experiments shows the interaction between VCL and NFASC is greatly reduced in SACS KO cells, despite NFASC being substantially overexpressed in SACS KO cells (Fig. 6d).

d. STRING protein interaction map depicting proteins identified across proteomics datasets. Lines between proteins indicate high confidence interactions (interaction score $>0.7$ ). We removed proteins with redundant interactions for clarity (for example most integrins have largely overlapping interactomes). Proteins identified in the SACS interactome profiling are circled, with the thick circle marking interactors identified in all replicates, thin circle marking interactors identified in a subset of samples. Proteins are colored by $\log _{2}$ fold change in proteome (left half) and cell surface proteome (right half). Striped lines indicate no detection. Clusters identified by k-means clustering are marked by grey background. 


\section{Supp. Fig. 1}

a

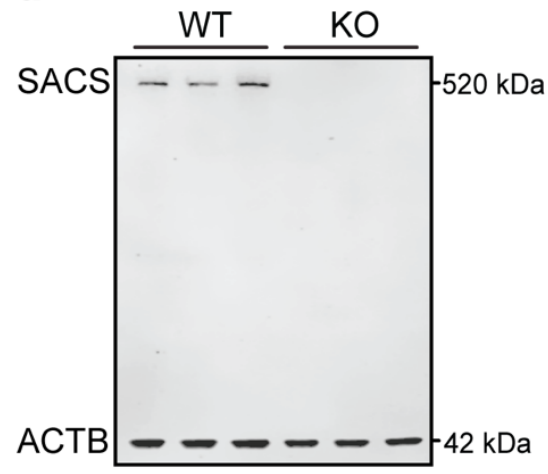

b

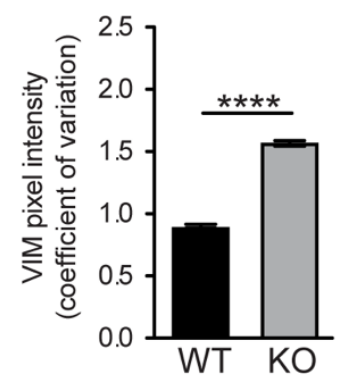

C

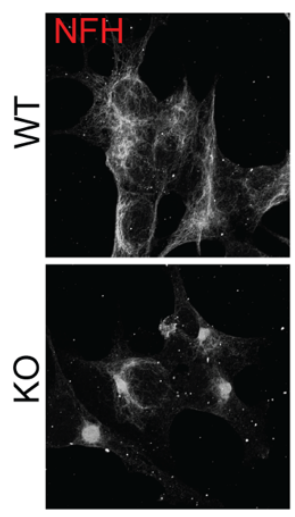

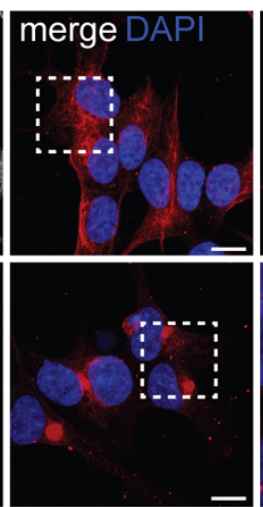

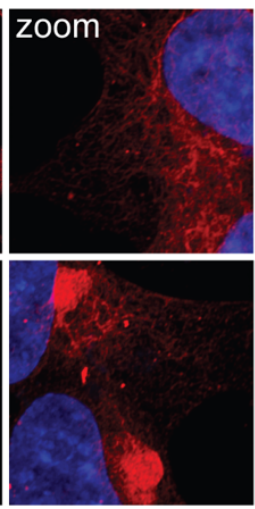

e

d
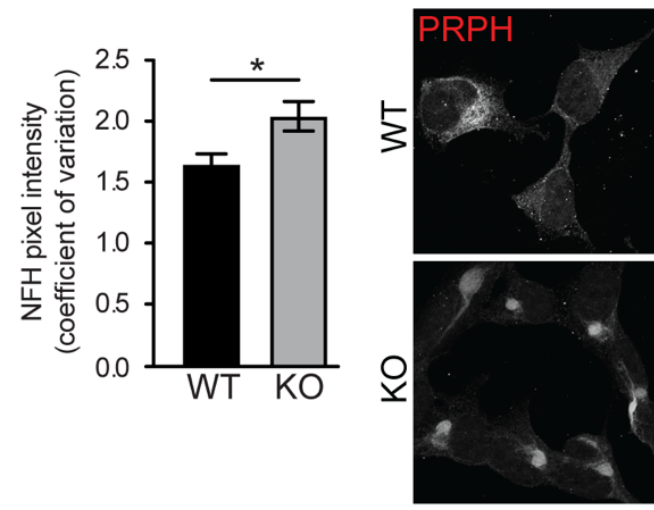

g

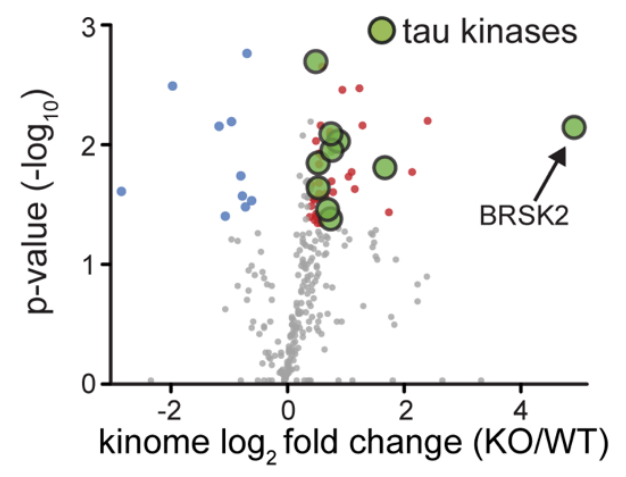

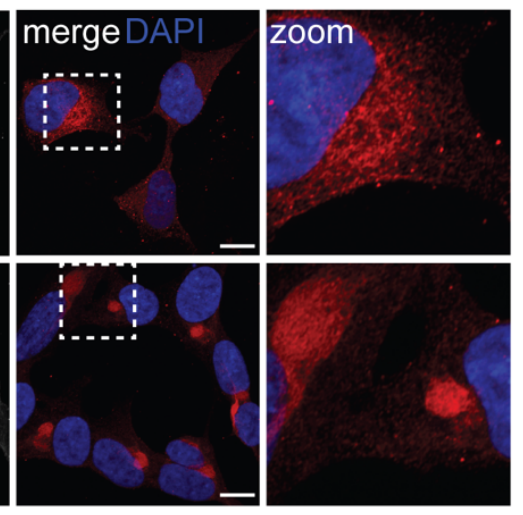

h

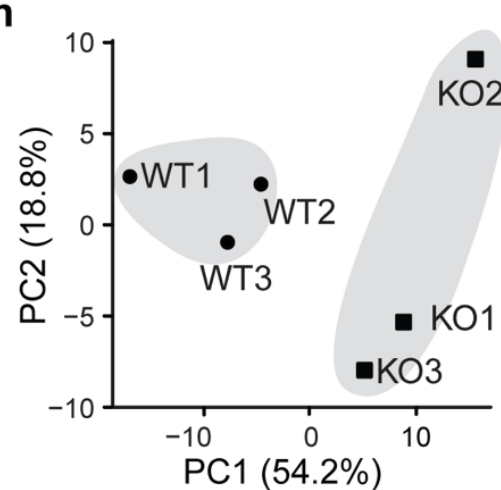

\section{Extended Data Figure 1 - SACS KO SH-SY5Y cells recapitulate cellular phenotypes consistent with} known deficits

a. Western blot for SACS and ACTB demonstrating the loss of SACS in SH-SY5Y KO cells.

b. Coefficient of variation of vimentin pixel intensity values across the cell, with lower values indicating uniform distribution and higher values indicating polarized protein distributions.

c. Representative confocal images of WT and SACS KO cells immunostained for the neurofilament heavy chain.

d. Coefficient of variation of NFH pixel intensity.

e. Representative confocal images of WT and SACS KO cells immunostained for peripherin, an intermediate filament protein found in neurons in the peripheral nervous system.

f. Phosphoproteomic analysis of SACS KO SH-SY5Y cells. Green circles mark specific phosphorylated residues on tau. 
g. Kinome profiling of SACS KO SH-SY5Y cells. Green circles mark kinases which are known to directly phosphorylate tau.

h. Principle component analysis of all kinases identified in kinome profiling data (Supp. Table 1). Unsupervised hierarchical clustering separated WT and KO cells (grey shading), suggesting widespread changes in the kinome of SACS KO cells. 
bioRxiv preprint doi: https://doi.org/10.1101/2021.08.20.456807; this version posted September 2, 2021. The copyright holder for this preprint

(which was not certified by peer review) is the author/funder, who has granted bioRxiv a license to display the preprint in perpetuity. It is made available under aCC-BY-NC-ND 4.0 International license.

\section{Supp. Fig. 2}

a

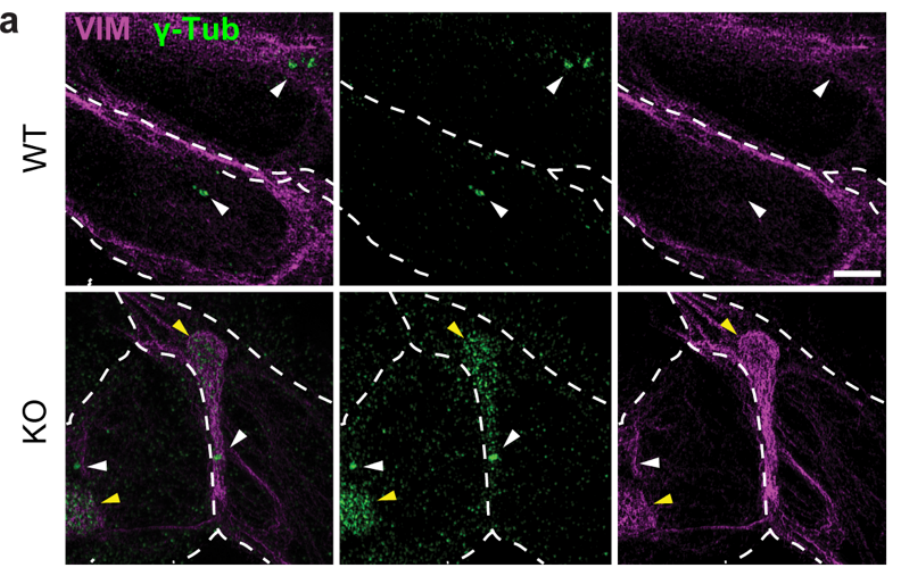

C
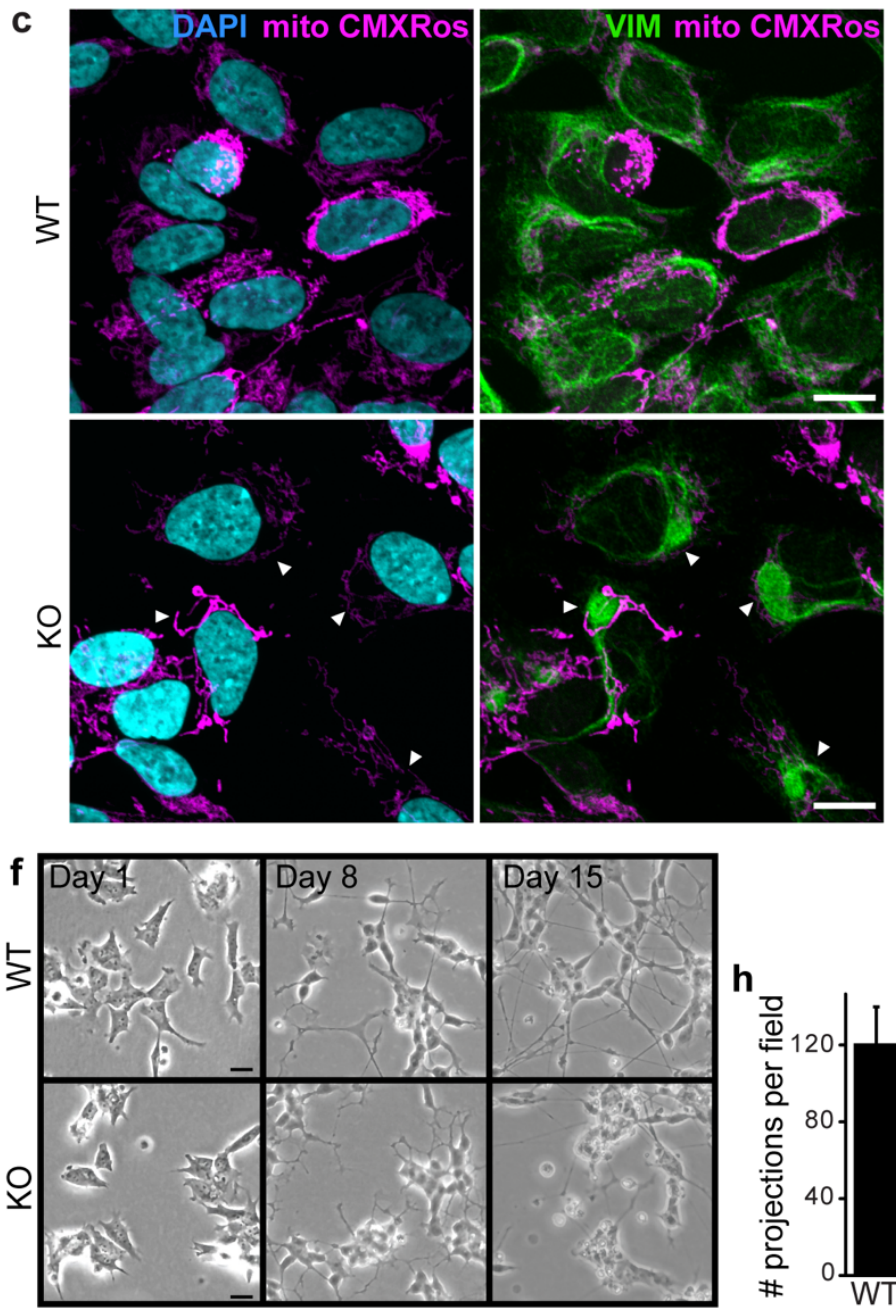

g MAP2

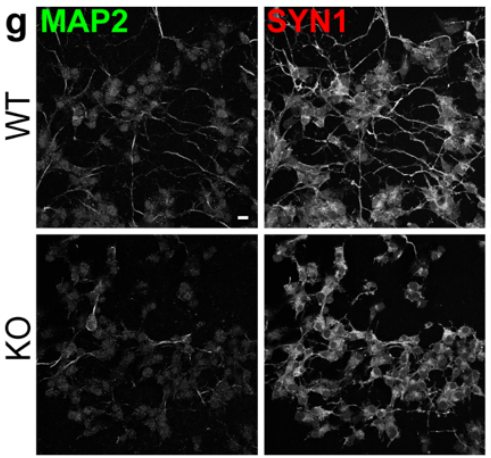

b

$\stackrel{\Upsilon}{x}$
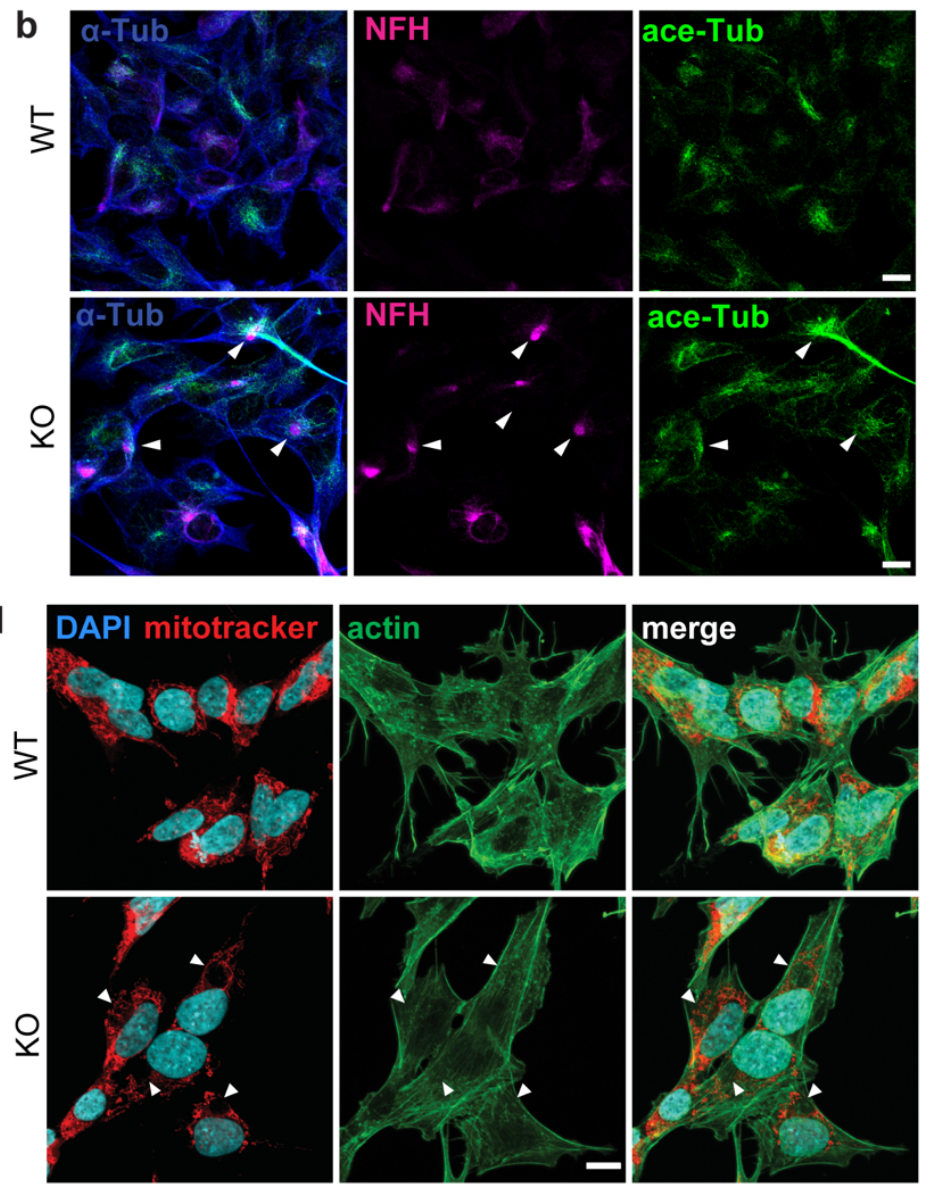

WT
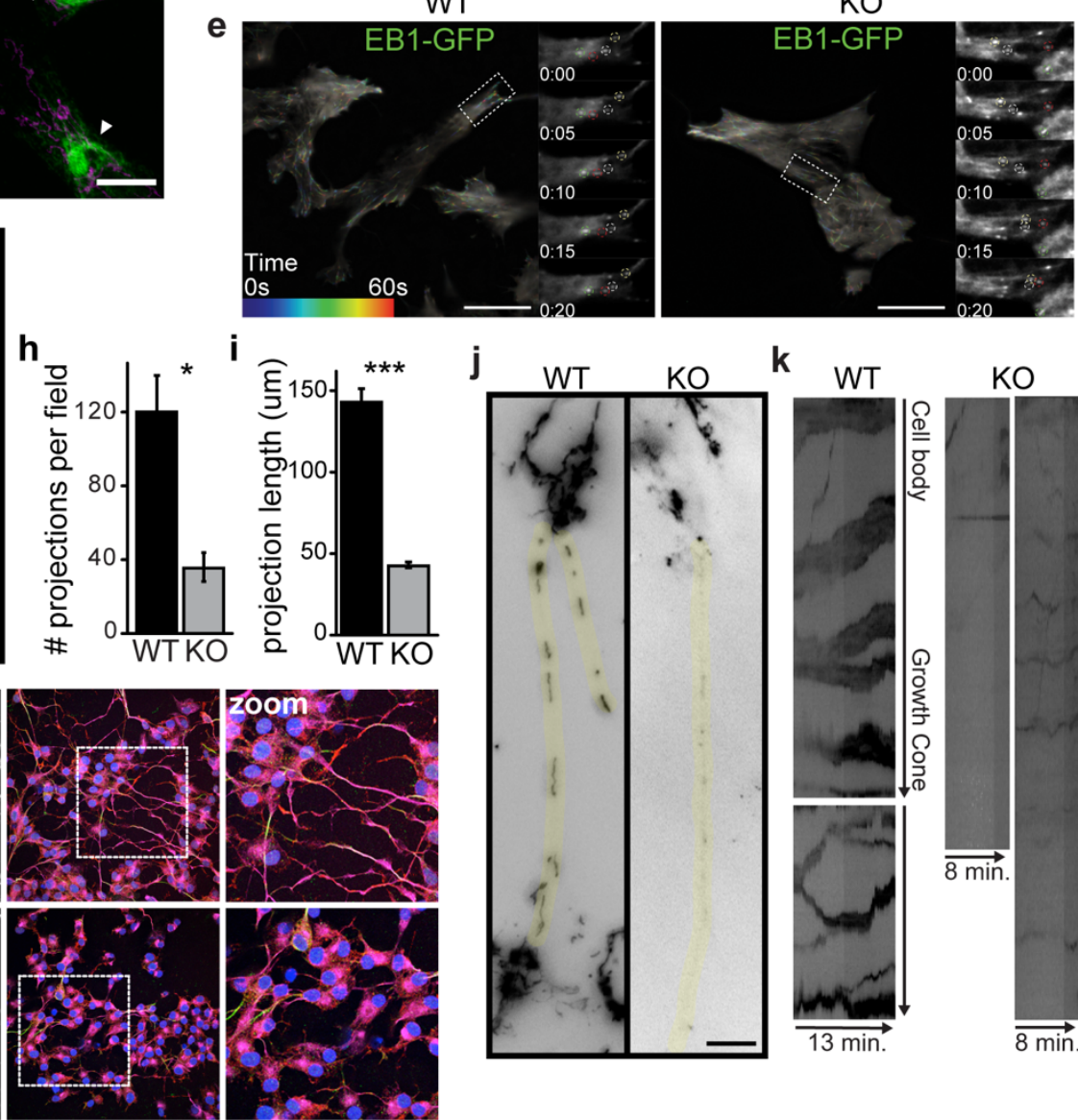
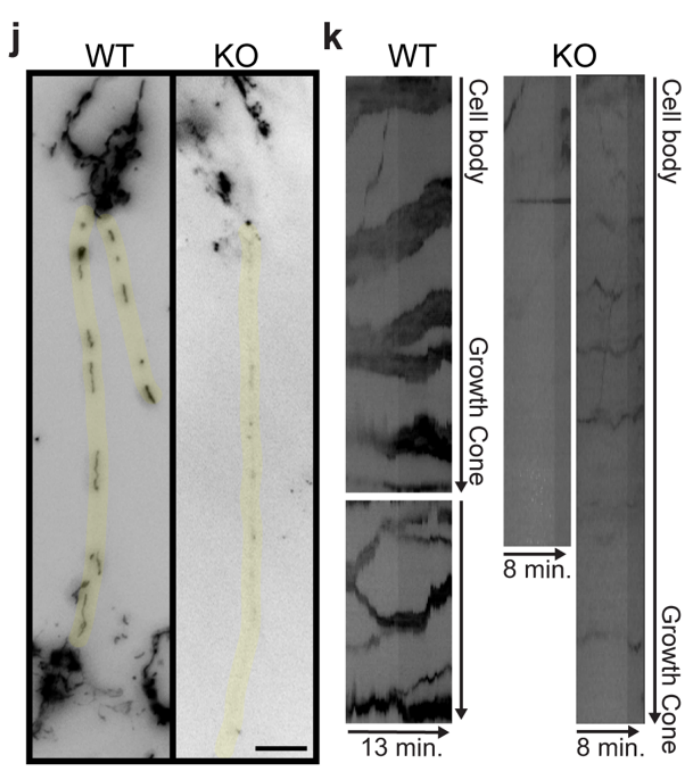


\section{Extended Data Figure 2 - Microtubule and mitochondria deficits in SACS KO cells}

a. Super resolution structural illumination microscopy images showing accumulation of gamma-tubulin within perinuclear vimentin bundles of SACS KO cells. White arrows point to centrioles, yellow arrowheads highlight the presence of gamma-tubulin within vimentin bundles in KO cells. Dashed white lines denote boundaries between adjacent cells. Scale bar $=1 \mathrm{um}$.

b. Representative confocal images of immunostaining for alpha tubulin, neurofilament heavy, and acetylated tubulin in WT and SACS KO cells. Arrowheads mark coincidence of acetylated tubulin and neurofilament bundles, suggesting that acetylated tubulin structures are found in proximity to neurofilament bundles, but also localize throughout the cell.

c. Representative confocal images of WT and SACS KO cells stained for the mitochondria membrane potential dependent dye CMXRos, vimentin, and nuclei (DAPI). Arrowheads highlight the exclusion of mitochondria from vimentin bundles.

d. Representative confocal images of WT and SACS KO cells immunostained for mitotracker, actin, and nuclei (DAPI). Arrowheads highlight the exclusion of mitochondria from vimentin bundles.

e. Representative TIRF microscopy images from WT and SACS KO cells expressing EB1-GFP. Microtubule growth tracks are color coded marking their position over time. Insets show the enlargement of outlined regions and movement of individual comet movement over time (circles), numbers refer to seconds.

f. Representative phase contrast brightfield images of WT and SACS KO cells across 15 days of neuronal differentiation.

g,h. Quantitation of the number of projections per field ( $\mathrm{g}$ ) and length of projection (h) of WT/KO cells demonstrating significantly reduced number and length of projections in SACS KO cells.

i. Confocal images of WT/KO cells after 15 days in differentiation conditions, stained for neuronal markers microtubule associated protein 2 (MAP2) and synapsin 1 (SYN1), and the intermediate filament protein nestin (NES), a marker of immature neurons. Scale bar $=10 \mu \mathrm{m}$.

j. Mitochondria labeled with mitoTracker GreenFM in neurites (highlighted in yellow) of 15 day differentiated WT/KO cells demonstrating the lack of elongated mitochondria in SACS KO neurites. Images were snapshots from live-cell time-lapse imaging.

k. Kymograph illustrating mitochondrial transport along neurites of differentiated WT/KO cells. Note that mitochondrial undergo both retrograde and anterograde movement in control but are relatively static in SACS KO cells. Scale bars $=10 \mu \mathrm{m}$. 


\section{Supp. Fig. 3}

a
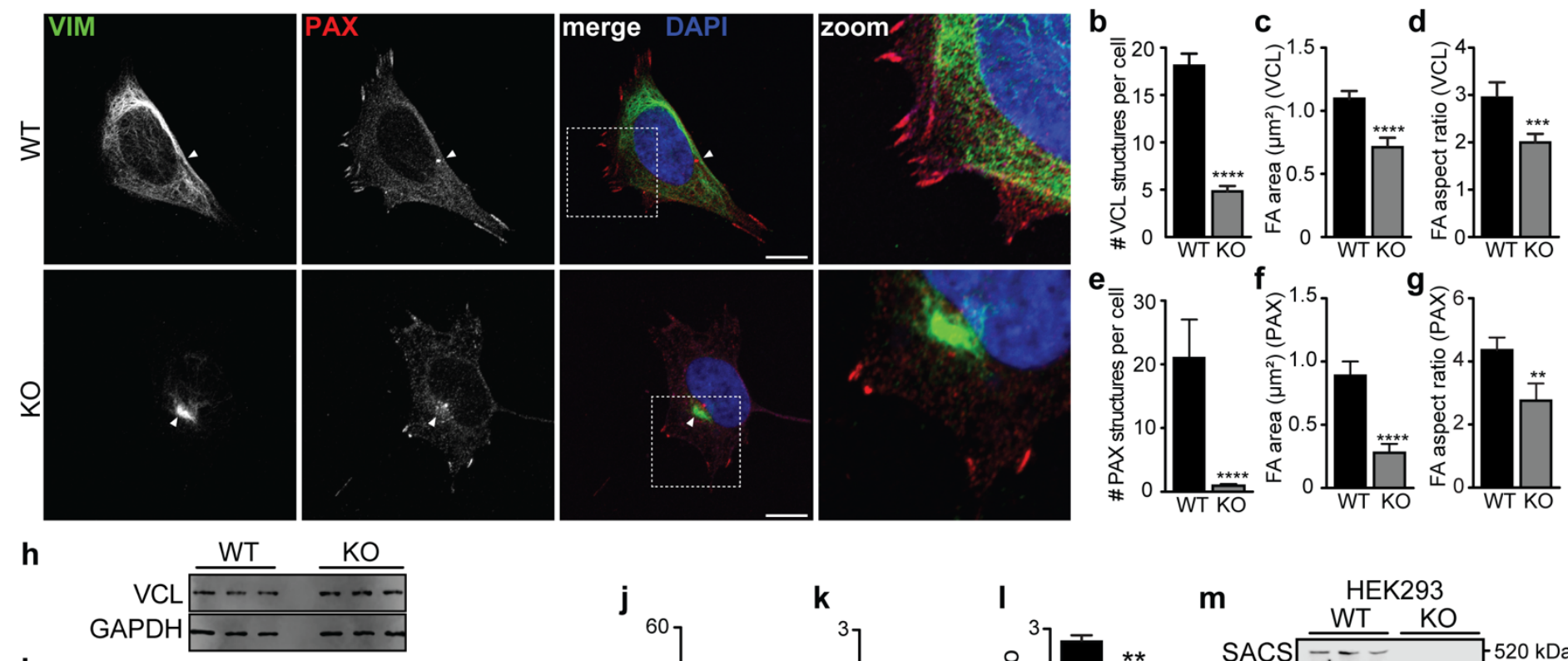

i
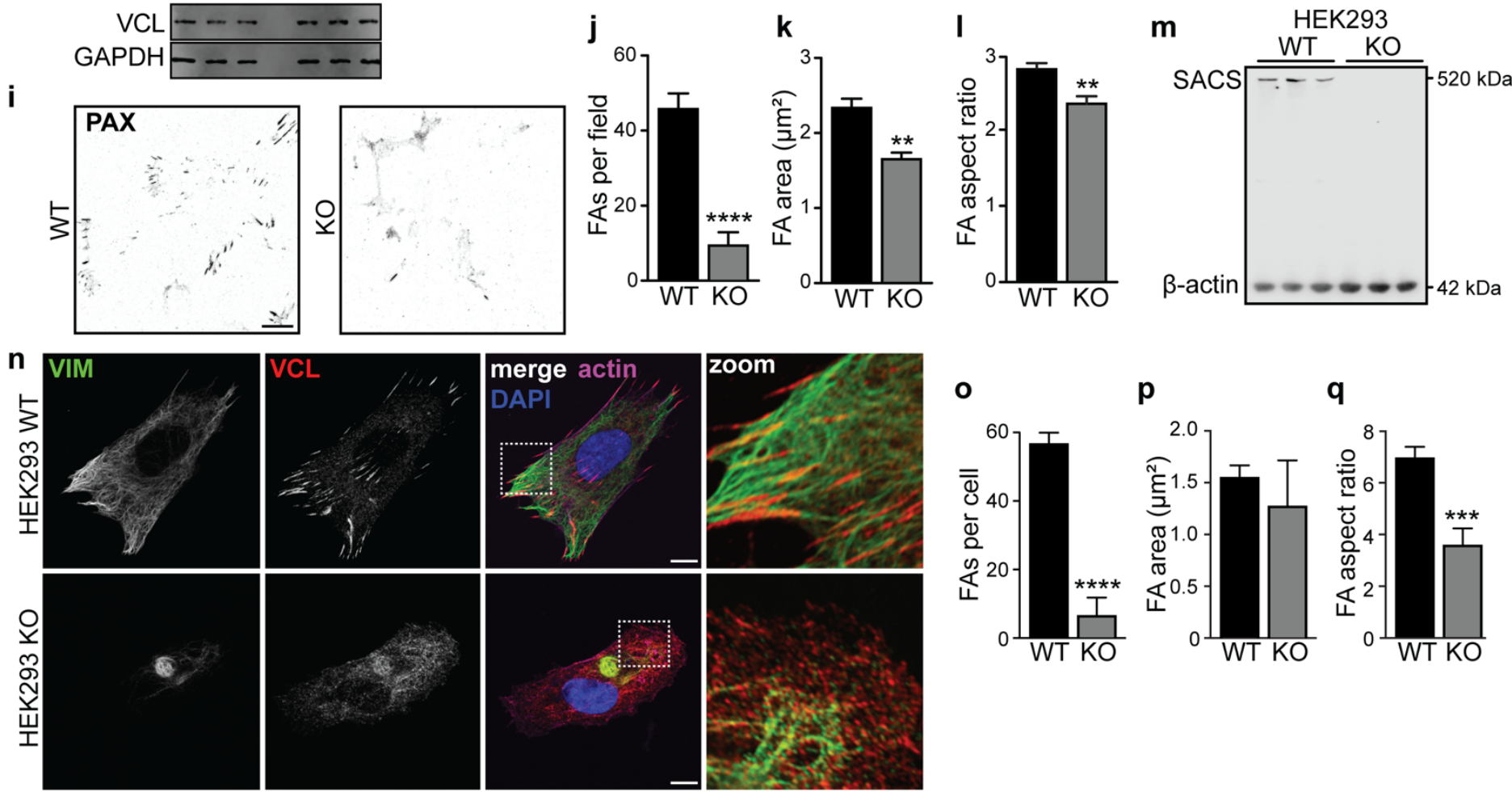

\section{Extended Data Figure 3 - Focal adhesions are disrupted in SACS KO cells}

a. Representative confocal image of WT/KO cells labelled with vimentin and paxillin. Arrowhead marks the PAX positive MTOC, which is sequestered in the vimentin bundle in SACS KO cells.

b-g. Quantification of images from Fig. 3c (b-d), and Extended Data Figure 3a (e-g). Aspect ratio = width:height ratio. $\mathrm{n}=3$ independent replicates.

h. Western blot for vinculin, showing that levels of the focal adhesion protein are unaltered in KO cells.

i. Representative image of cover slips treated with hypotonic shock to remove cell bodies, leaving focal adhesions retained through ECM interaction.

j-I. Quantification of the incidence, area, and aspect ratio of paxillin positive focal adhesions in WT/KO cells.

m. Western blot for SACS and ACTB demonstrating the loss of SACS in HEK293 KO cells.

n. Confocal images of HEK293 cells immunolabeled for vimentin, vinculin, and actin. 
bioRxiv preprint doi: https://doi.org/10.1101/2021.08.20.456807; this version posted September 2, 2021. The copyright holder for this preprint

(which was not certified by peer review) is the author/funder, who has granted bioRxiv a license to display the preprint in perpetuity. It is made available under aCC-BY-NC-ND 4.0 International license.

o-q. Quantification of images from Supp. Fig. 3n, suggesting focal adhesion deficits are consistent with $\mathrm{SH}$ SY5Y cells.

r. Representative confocal image of WT/KO SH-SY5Y cells immunolabelled for ITGA6 and ITGB1. 


\section{Supp. Fig. 4}

a b

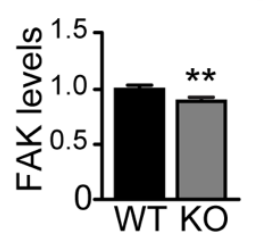

e $\quad f$

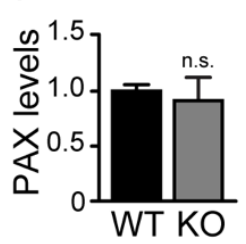

i

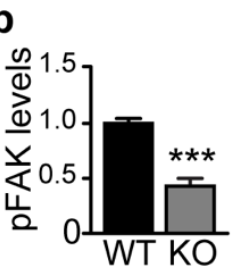

f

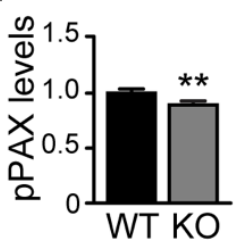

C

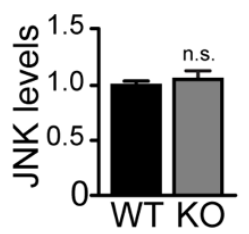

g

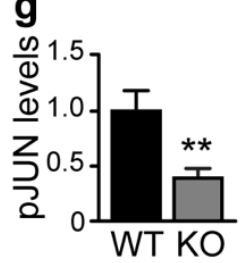

d

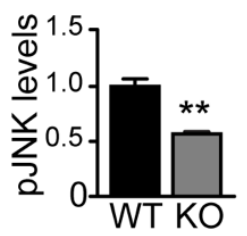

h

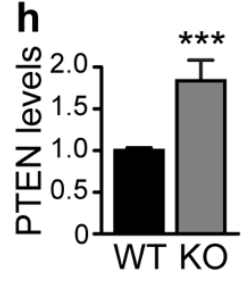

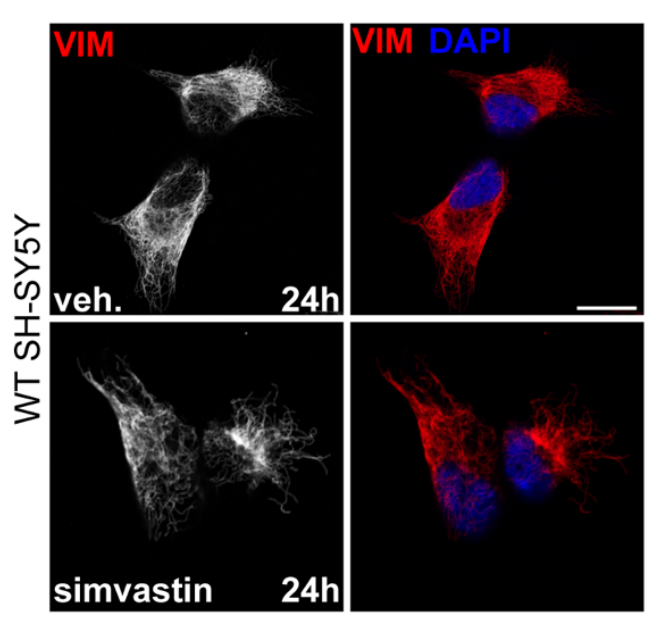

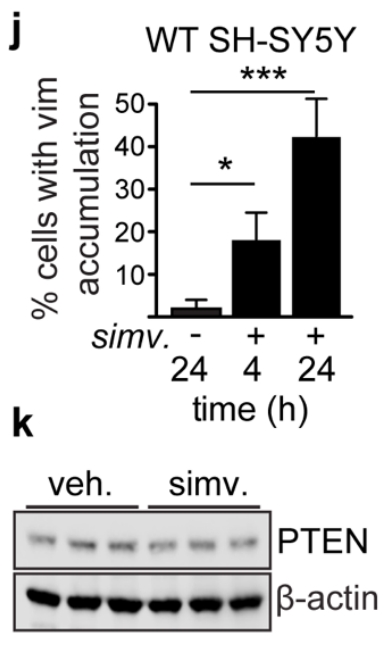

I

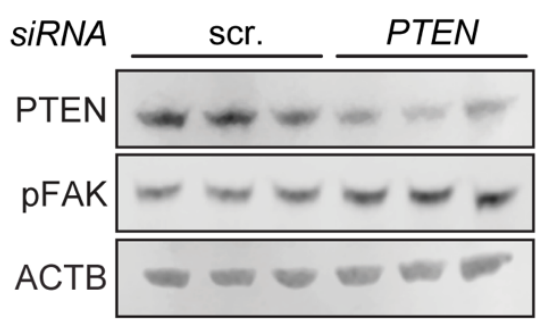

m

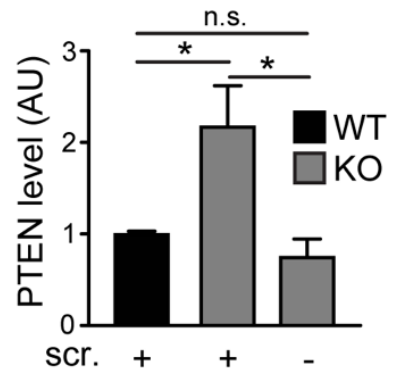

n

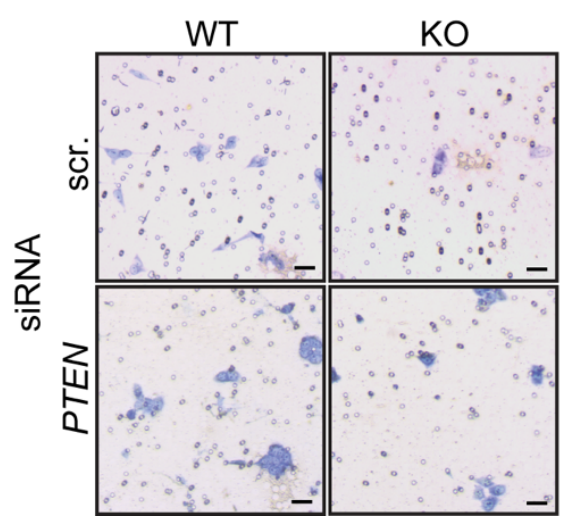

0

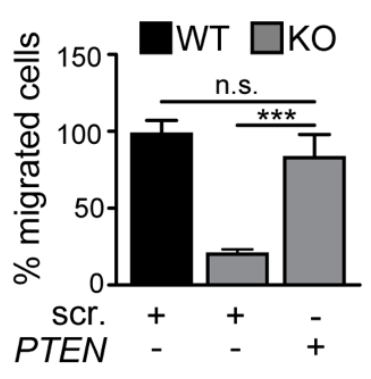

Extended Data Figure 4 - Modulating PTEN rescues cellular phenotypes in SACS KO cells

a-h. Quantification of immunoblots from Fig. 4c. Intensity normalized to ACTB. $n=3$ biological replicates.

i. Representative confocal images of the induction of vimentin bundling by simvastin. Scale bars $=10 \mu \mathrm{m}$. 
j. Quantification of vimentin bundling phenotype induced by simvastin over time.

k. Western blot of PTEN levels in 24 hour simvastin treated WT cells, suggesting that vimentin bundling does not affect PTEN levels.

I. Representative Western blot of SACS KO cells treated with siRNAs targeting PTEN or scrambled.

$\mathrm{m}$. Quantification of PTEN levels in WT/KO cells treated with scrambled or PTEN targeting siRNAs, suggesting PTEN is returned to WT levels in SACS KO cells. $\mathrm{n}=3$.

n. Representative images of WT/KO cells plated in Transwell chambers with $8 \mu \mathrm{m}$ pores, allowed 24 hours for cells to migrate through pores, and fixed and stained with Giemsa blue. Scale bar $=20 \mu \mathrm{m}$.

o. Quantification of transwell assays in WT/KO cells treated with the indicated siRNAs. $n=5$ per cell line. 
bioRxiv preprint doi: https://doi.org/10.1101/2021.08.20.456807; this version posted September 2, 2021. The copyright holder for this preprint

(which was not certified by peer review) is the author/funder, who has granted bioRxiv a license to display the preprint in perpetuity. It is made available under aCC-BY-NC-ND 4.0 International license.

\section{Supp. Fig. 5}

a

hyperphosphorylated proteins

nuclear matrix microtubule

intracellular signal transduction RNA binding postsynapse

protein serine kinase activity transcription coregulator activity microtubule polymerization cell-cell contact zone

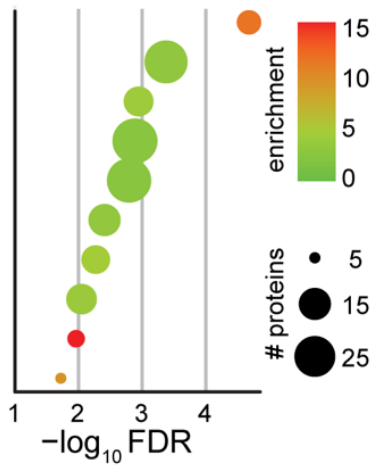

d

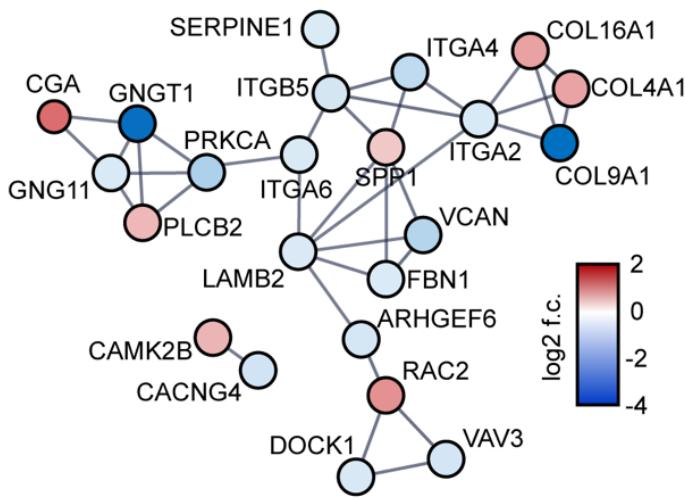

g

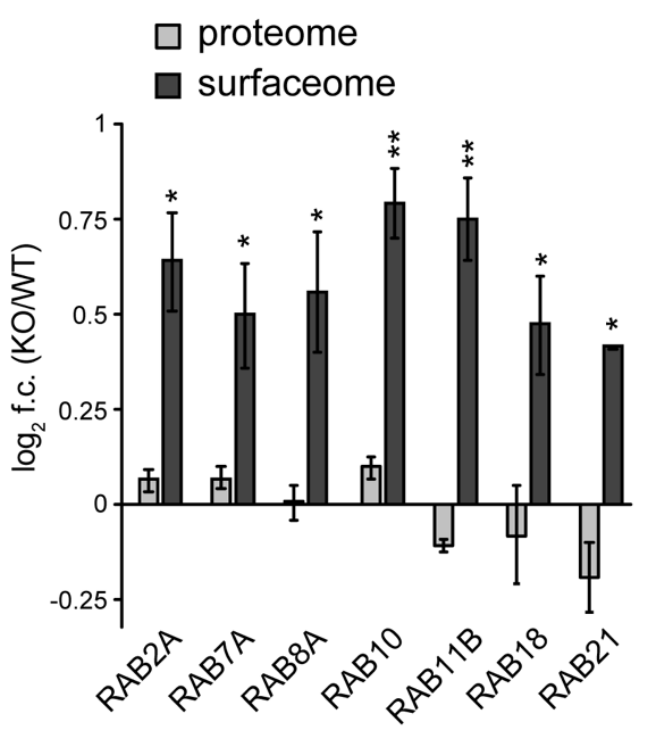

b

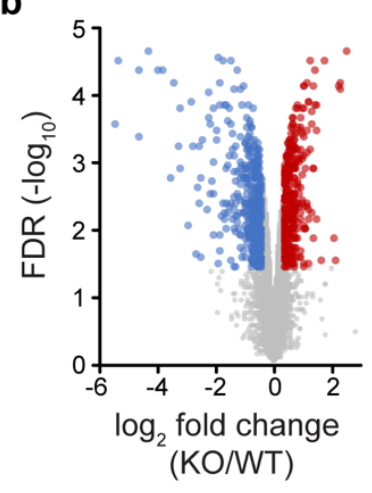

C

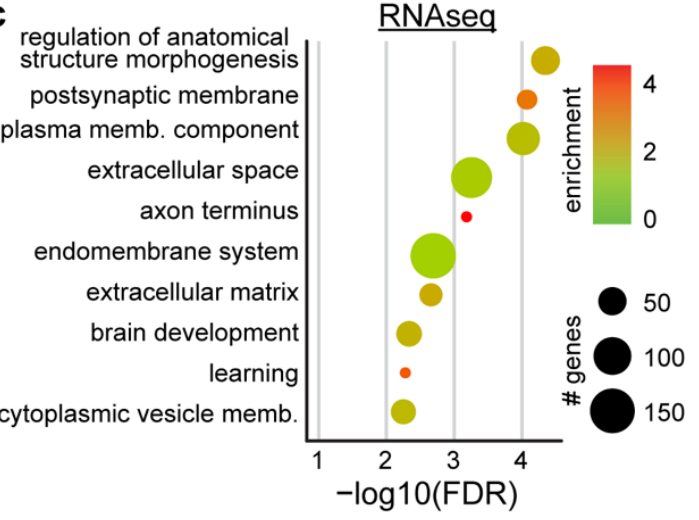

f

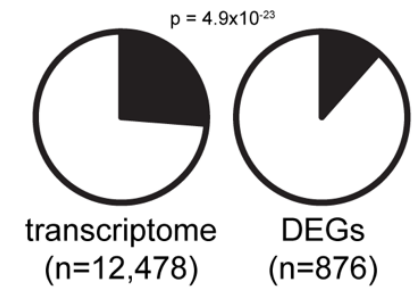

detected in proteome \& transcriptome $\square$ detected only in transcriptome 6194 proteins detected

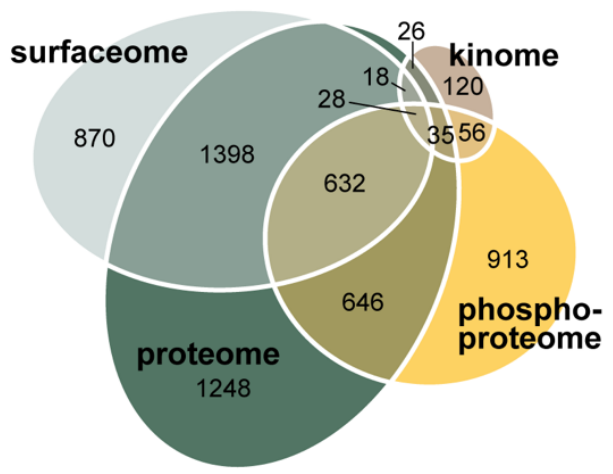

h
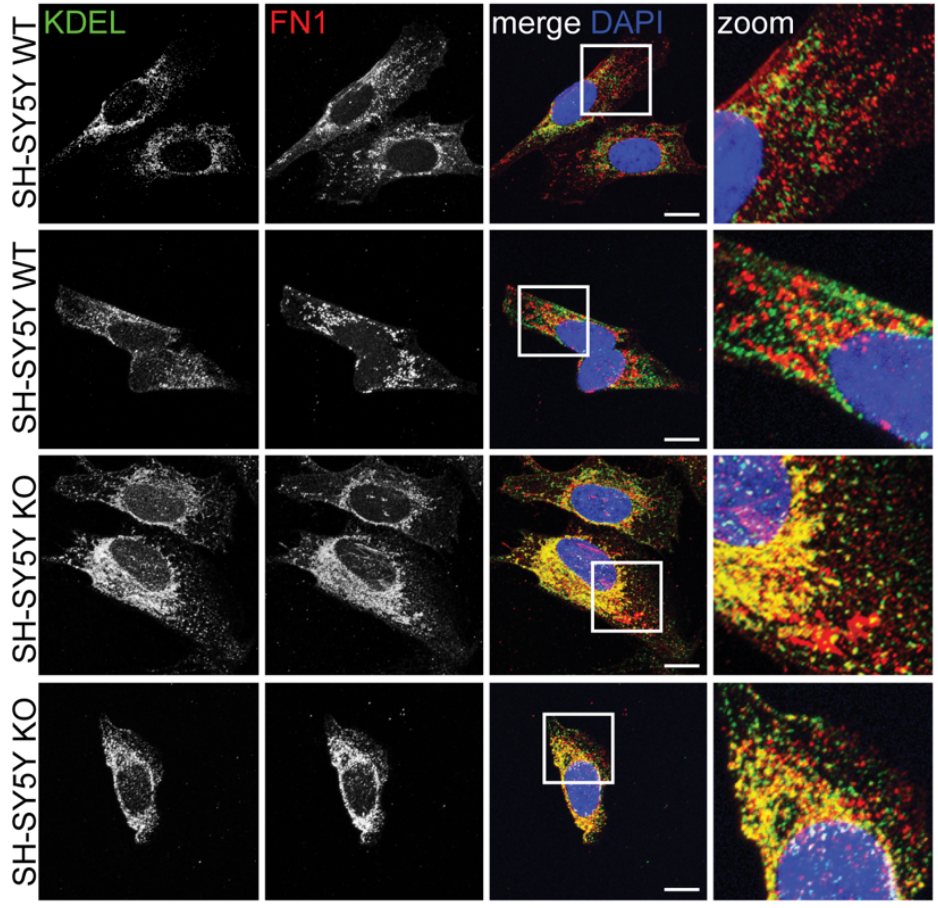

\section{Extended Data Figure 5 - Altered transcription of synaptic adhesion and vesicular proteins}

a. GO term analysis of hyperphosphorylated proteins in proteomic analysis $\left(p<0.05, \log _{2}\right.$ fold change $-/+$ 0.4 ) (Extended Data Fig. 1f), identifying multiple terms related to nuclear/transcriptional processes.

b. RNAseq of 15 day neuronally differentiated SH-SY5Y cells. 
c. GO term analysis of differentially expressed genes suggests that synaptic and vesicular transport genes are altered in neurons $\left(p<0.05, \log _{2}\right.$ fold change $\left.-/+0.5\right)$.

d. Interaction network of cell adhesion proteins that are differentially expressed.

e. Overlapping gene/protein identification from RNAseq and proteomics, showing that DEGs were not detected as readily as genes in the rest of the transcriptome. Hypergeometric test was used to calculate enrichment $p$-value.

f. Euler diagram of protein identification across all mass-spec datasets.

g. $\log _{2}$ fold change of Rab proteins in proteome and surfaceome datasets. Asterisks refer to statistical significance in each dataset. No Rabs were significant in the proteome.

h. Representative confocal images of cells immunolabelled for fibronectin and KDEL in in WT/KO SHSY5Y cells. 
bioRxiv preprint doi: https://doi.org/10.1101/2021.08.20.456807; this version posted September 2, 2021. The copyright holder for this preprint (which was not certified by peer review) is the author/funder, who has granted bioRxiv a license to display the preprint in perpetuity. It is made available under aCC-BY-NC-ND 4.0 International license.

\section{Supp. Fig. 6}

a

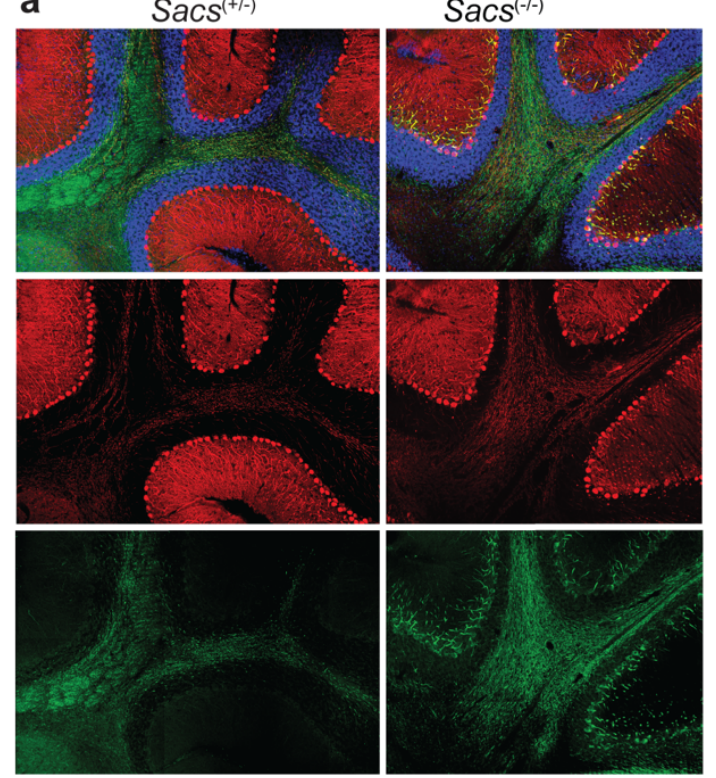

\section{ITGA1 CALB1 NEUN}

C
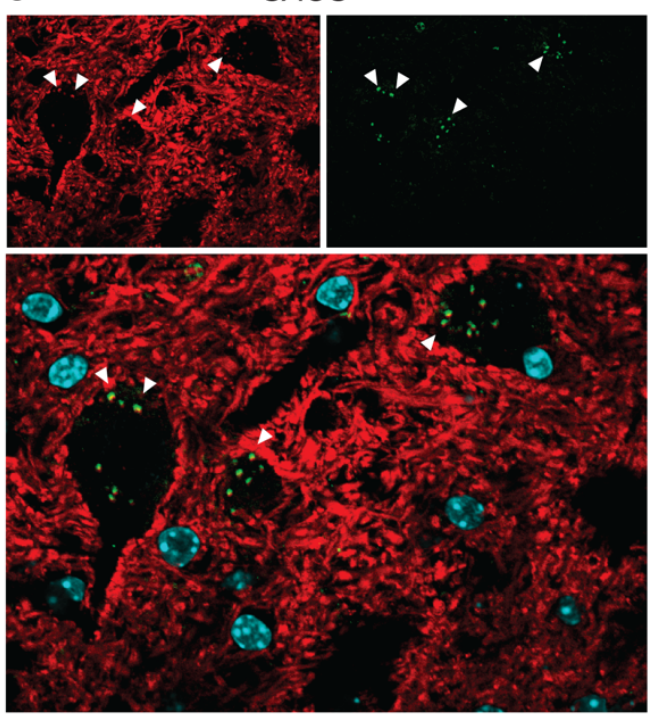

PAX SYN1 DAPI b
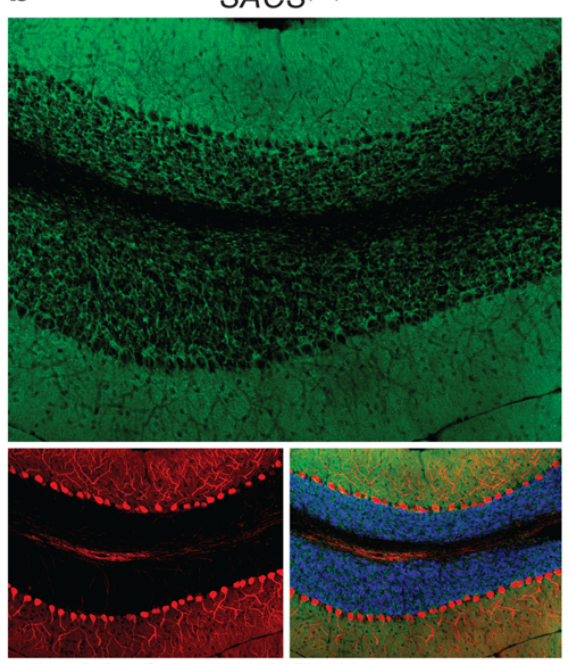

NFASC CALB1 NEUN

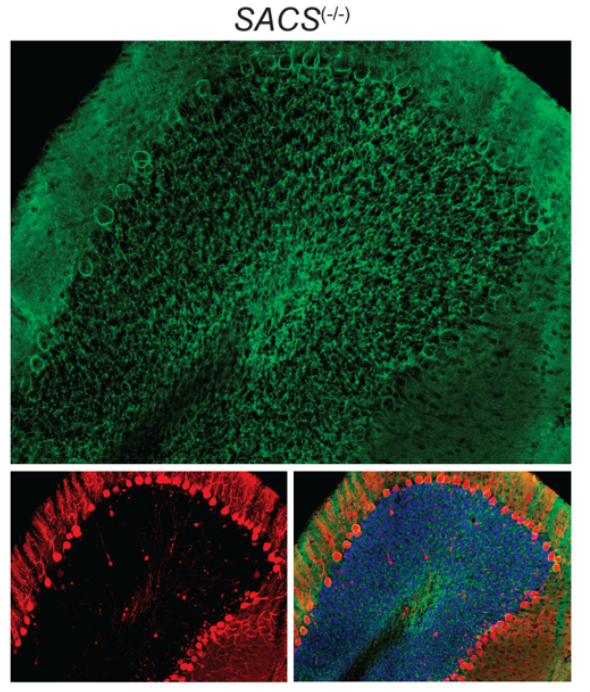

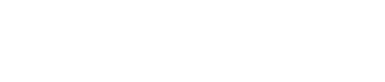
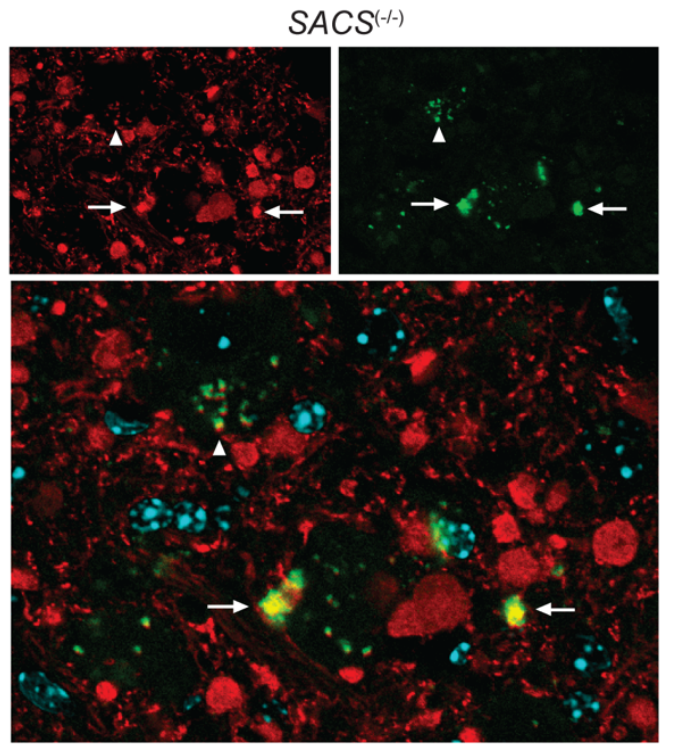

\section{Extended Data Figure 6 - Cerebellar imaging in SACS KO mice}

a. Widefield view of ITGA1 staining in Sacs $^{(+/)}$and Sacs ${ }^{(-)}$mice. ITGA1 staining is normally observed along Purkinje neuron axon tracts, but is mislocalized to the proximal dendritic arbor in Sacs ${ }^{(--)}$mice.

b. Widefield view of NFASC expression in dorsal cerebellar lobules in P120 mice, demonstrating increased NFASC intensity surrounding Purkinje neuron soma in Sacs ${ }^{(-)}$mice.

c. Representative confocal image of neurons (black voids) in the DCN. Arrowheads mark colocalization between paxillin and synapsin, arrows mark large extracellular PAX+/SYN+ aggregates. 
bioRxiv preprint doi: https://doi.org/10.1101/2021.08.20.456807; this version posted September 2, 2021. The copyright holder for this preprint (which was not certified by peer review) is the author/funder, who has granted bioRxiv a license to display the preprint in perpetuity. It is made available under aCC-BY-NC-ND 4.0 International license.

\section{Supp. Fig. 7}
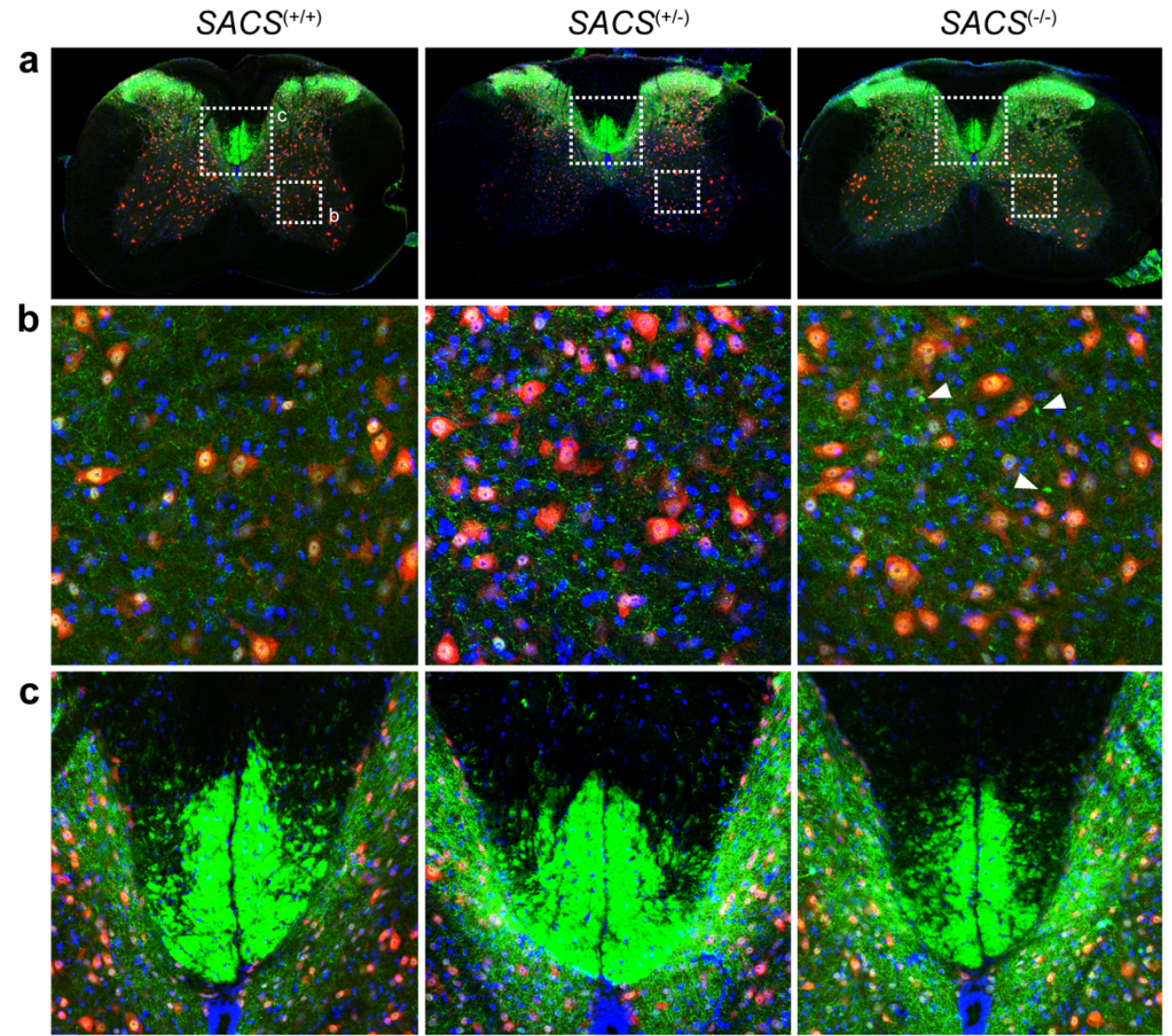

b
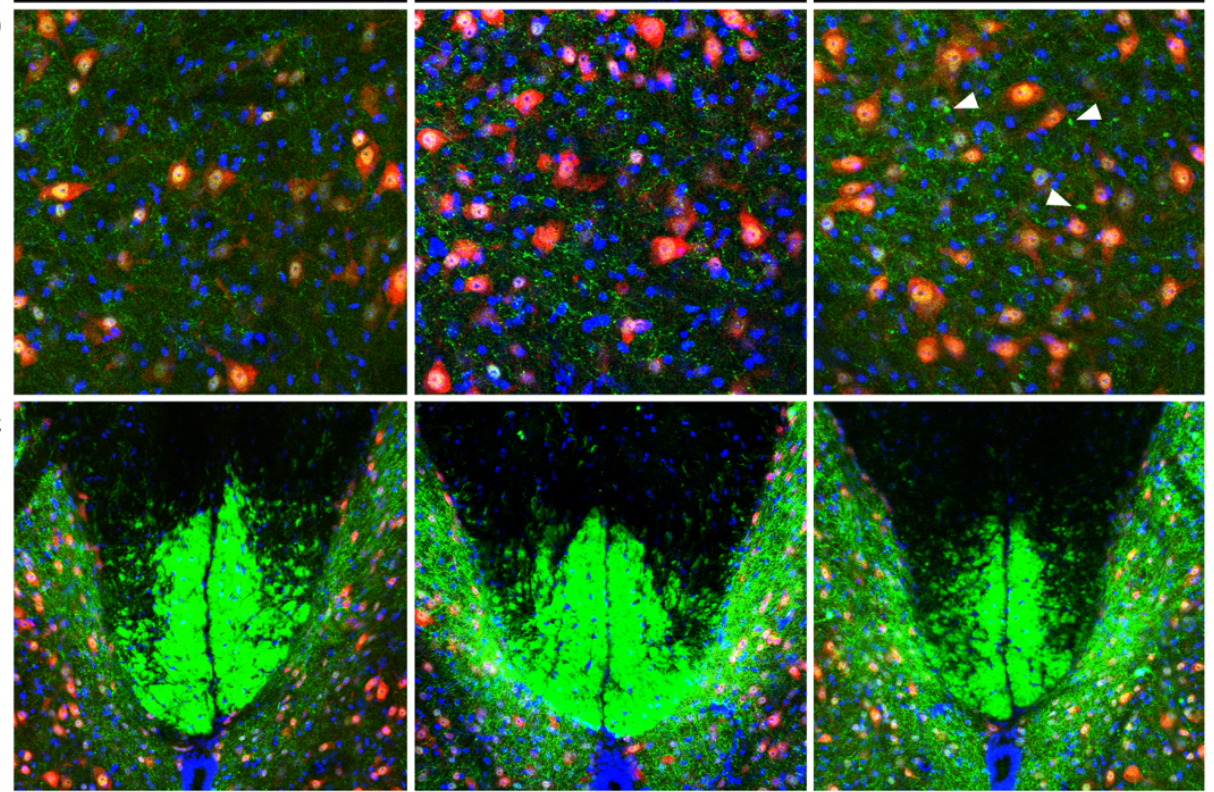

NeuN PRKCg DAPI

\section{Extended Data Figure 7 - Axonal and synaptic alterations in spinal cord from P120 SACS KO mice}

a. Representative confocal images of the lumbar spinal cord labeled for PRKCg (a neuronal marker specific to cortical layer 5 neurons ${ }^{82}$ ) NEUN, and DAPI in WT, SACS ${ }^{(+/)}$, and $S A C S^{(-/)}$mice.

b. High magnification of area of ventral spinal cord indicated in panel a. White arrowheads mark abnormal axonal swellings, similar in appearance to those surrounding DCN neurons.

c. High magnification of lumbar the corticospinal tract indicated in panel a, showing an overall decrease in axonal tract density in $S A C S^{(-)}$mice. 


\section{Supp. Fig. 8}

a
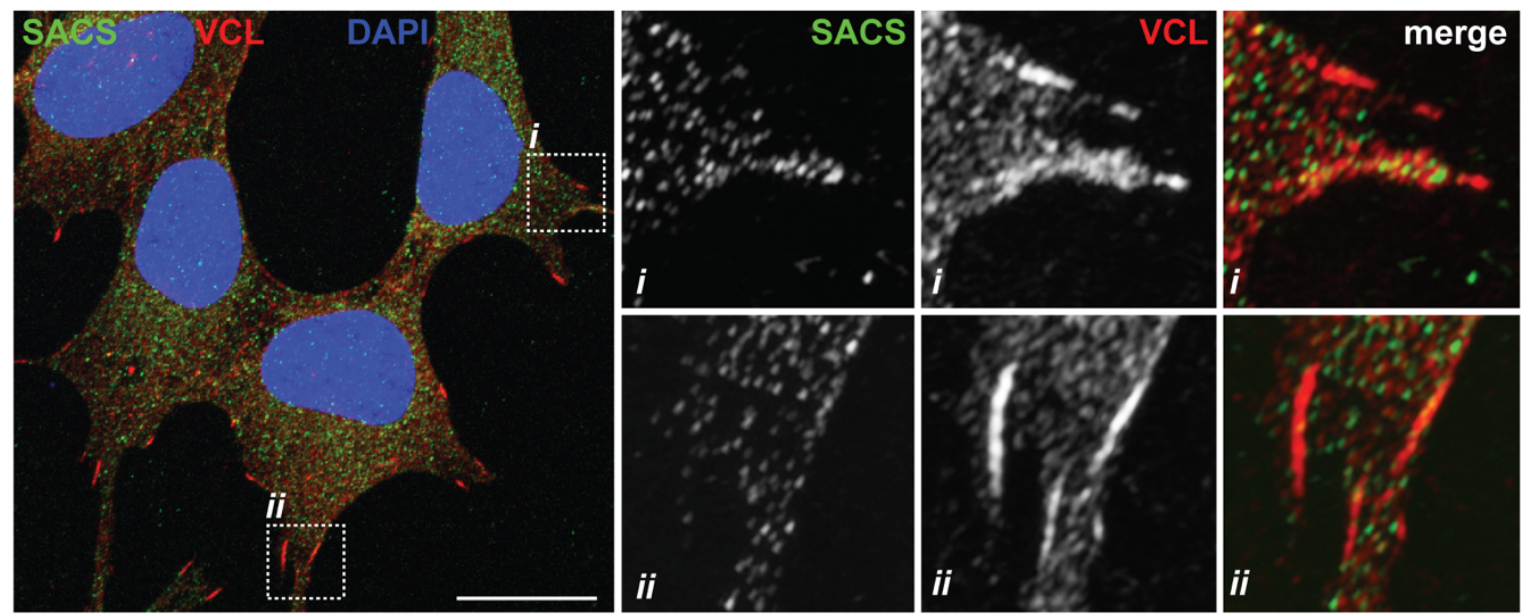

b
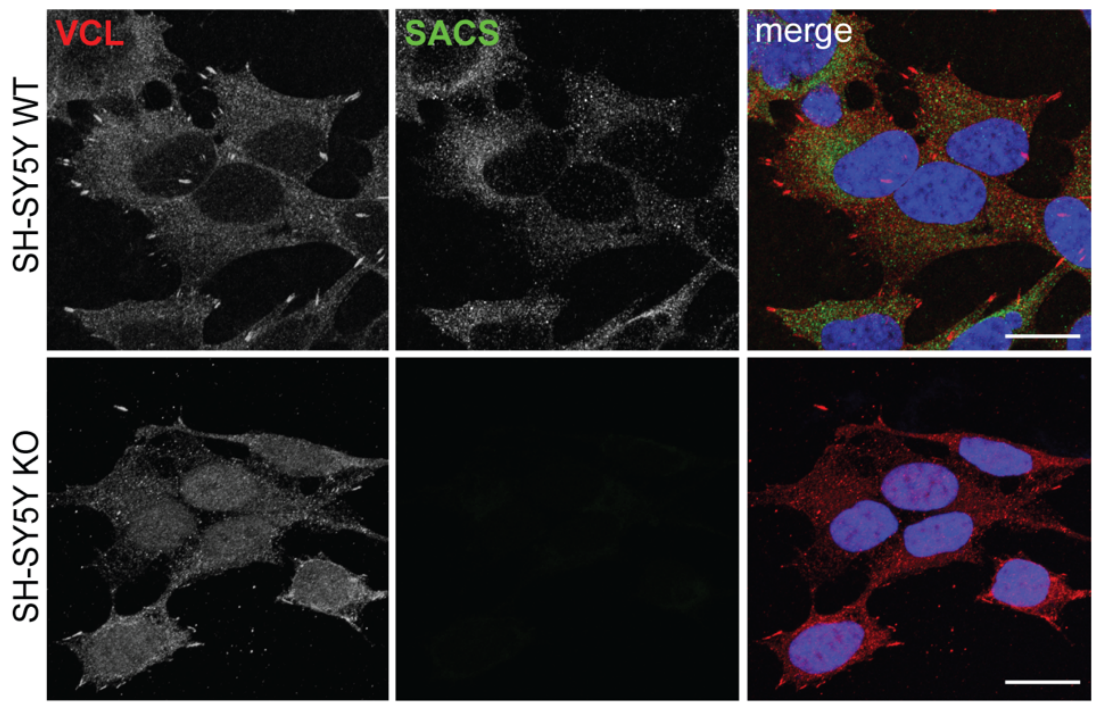

C

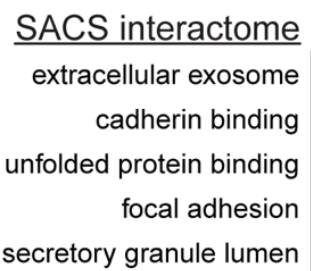

neg. regulation of apoptotic process

ficolin-1-rich granule lumen double-stranded RNA binding

nuclear matrix melanosome

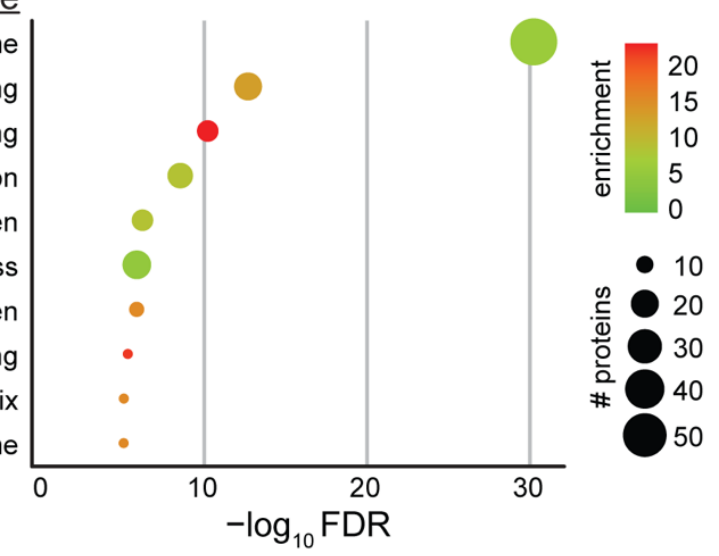

\section{Extended Data Figure 8 - SACS interactors}

a. Representative confocal image for sacsin and vinculin in WT SH-SY5Y cells demonstrating SACS colocalizes with focal adhesions.

b. Representative confocal image for SACS KO cells processed in parallel to (a), demonstrating the specificity of SACS staining.

c. GO term analysis of all proteins identified in the SACS co-IP interactome (Supplementary Table 4). 